\title{
Time-dependent and Energy Dissipation Effects on the Electro-Mechanical Response of PZTs
}

\author{
Kamran A. Khan, ${ }^{1 *}$ Anastasia H. Muliana, ${ }^{2}$ \\ Hassene Ben Atitallah ${ }^{3}$ and Zoubeida Ounaies ${ }^{3}$ \\ ${ }^{1}$ Department of Aerospace Engineering, Khalifa University of Science, Technology and \\ Research (KUSTAR), Abu Dhabi, UAE \\ ${ }^{2}$ Department of Mechanical Engineering, Texas A\&M University \\ ${ }^{3}$ Department of Mechanical and Nuclear Engineering, the Pennsylvania State \\ University \\ *Corresponding author: kamran.khan@ kustar.ac.ae
}

\begin{abstract}
In this study, we formulate a constitutive model for coupled thermo-electro-mechanical behavior of piezoelectric ceramics, i.e. lead zirconate titanate (PZT) that takes into account time-dependent behavior and heat generation due to energy dissipation from the electro-mechanical response. Experimental studies show that PZTs dissipate energy when subjected to cyclic electric fields. The dissipated energy is, in some part, converted into heat and raises the temperature of the PZTs. The hysteretic dielectric and strain responses are also dependent on the amplitude and frequency of the applied electric field. The aim of this study is to investigate the energy dissipation and time-dependent effects on the electro-mechanical responses of polarized PZTs. The thermodynamics of irreversible processes for modeling dissipative material responses is adopted to incorporate the time-dependent electromechanical coupling effect. A Gibbs free energy is defined in order to account for the different time-dependent and dissipation behaviors in piezoelectric materials under combined electro-mechanical stimuli. Experimental data on polarized PZTs and PZT based composites at applied electric field magnitudes lower than the coercive electric field, are used to examine the model. The constitutive equations along with the energy equations are solved numerically and used to predict the creep, hysteretic and heat generation responses of PZTs and composites at different frequencies and cyclic electric fields.
\end{abstract}

Keywords Piezoelectric, Electro-mechanical, Time-dependent, Dissipation, Heat Generation, PZT, AFC. 


\section{Introduction}

Ferroelectric ceramics like PZTs have been widely used in actuators, sensors, energy harvesting devices, vibration suppression, structural health monitoring systems (Lines and Glass, 1977). During their service, ferroelectric materials are often exposed to various external stimuli such as electrical, mechanical and thermal stimuli and can exhibit a strong coupling between mechanical and other effects, which depend on the magnitude and rate of the external stimuli (Uchino, 2010). Ferroelectric ceramics also show rate-dependent (or frequency-dependent) hysteretic electro-mechanical response and dissipate energy under cyclic electric field (Ben Atitallah et al., 2016; Pritchard et al., 2004). The energy dissipation causes temperature increase and can alter the electromechanical properties of ferroelectric materials, and thus affecting their performance. In order to better predict life performance of ferroelectric materials, it is necessary to understand their time-dependent hysteretic responses and dissipation mechanisms under different external stimuli. In this study, we focus on understanding the linear hysteresis response of polarized ferroelectric materials under electrical loading (below coercive field to avoid polarization switching) at off-resonant frequencies and investigate the amount of heat generation.

Several experimental and theoretical studies have been conducted to understand hysteretic and time dependent response of PZTs. Most of these studies have been done on polarization switching behavior, e.g. (Schmidt, 1981), (Li and Weng, 1999), (Muliana, 2011). Hysteretic responses are also seen in polarized ferroelectric materials under relatively low electric amplitude (minor loops ${ }^{1}$ ) in which polarization switching does not occur (Ben Atitallah et al., 2010). When subjected to cyclic electric fields, the piezoelectric ceramics show hysteretic responses, which strongly depend on the amplitude and frequency of electric field, existence of

\footnotetext{
${ }^{1}$ In minor loops, the hysteretic strain shows an ellipsoidal shape or distorted ellipsoidal shape, while when polarization switching occurs butterfly hysteretic strain is seen.
} 
stresses, and ambient temperatures ((Fett and Thun, 1998), (Liu, 2011)). Zhou and Kamlah (2006) investigated the time dependent effects of a commercial soft piezoelectric ceramics under constant electric field, cyclic electric field and stresses at room temperature. The time dependent hysteretic effects were found to be significant at higher stresses and at electric field near the coercive electric field.

Heat generation is one of the major issues in piezoelectrics when subjected to cyclic electric fields. Several systematic studies were conducted to analyze the loss mechanisms in piezoelectric materials for high power density applications (Uchino and Hirose, 2001). Heat generation in various PZT based actuators were studied under a small electric field applied at resonant frequency (Zheng et al., 1996) and under a large electric field applied at off-resonance frequency (Tashiro et al., 1997). The heat generation at resonance frequency was attributed mainly to the mechanical loss while polarization - electric field hysteresis loss were the main reason for heat generation at off-resonance electrical loadings (Uchino, 2010). Further discussion on dielectric behaviors of piezoelectric material can be found in (Ye, 2008). Recently, Ben Atitallah et al. (2016) studied the hysteretic response of PZT fiber and active fiber composites (AFC) at several frequencies and different electric field magnitudes. At relatively low electric field magnitude the responses were linear. For the electric field loading around 10 minutes, they observed substantial temperature rise in the sample. However, the systematic temperature changes in the material were not recorded during the tests. The present study investigates the temperature changes due to cyclic electric field. We hypothesize that this temperature increase is due to the dissipation of energy and can cause changes in the response of the ferroelectrics ceramics. Our aim is to develop a constitutive model for time-dependent electro-mechanical responses of piezoelectric ceramics, such as PZTs, incorporating the heat generation due to dissipation of energy. 
Piezoelectric ceramics, such as PZT, are brittle, can only undergo relatively small strains, and experience time-dependent behaviors when subjected to electro-mechanical stimuli. Understanding coupled electro-thermo-mechanical response in PZT that takes into accounts the dissipation of energy is currently limited. Chen (2009) derived a thermo-electro-viscoelastic constitutive equation based on time-integral model that incorporates heat generation due to the dissipation of energy and damage. The theoretical formulation was presented for general large deformation responses without showing any application of the model. In this paper, we adopt the thermodynamics of irreversible processes (TIP) and modify the approach used for viscoelastic responses (Schapery, 1997; Khan and Muliana, 2012), in order to model the thermo-electromechanical responses of piezoelectric materials. A Gibbs free energy is defined to obtain the constitutive relations and energy equation capable of incorporating temperature evolution in the materials with time-dependent electro-thermo-mechanical responses. The model is currently restricted to linear time-dependent responses.

This paper is organized as follows. Section 2 describes the formulation of a coupled electrothermo-mechanical model. Generalized constitutive relations and energy equation in terms of dissipation potentials are obtained from the energy balance and the entropy production inequality. A Gibbs free energy function is proposed to account for different dissipation effect under combined electro-mechanical stimuli. Specific forms of the constitutive equations, the evolution equation for each dissipation mechanism, and energy equations are presented. Section 3 presents experimental tests on PZT5A fibers and active fiber composite (AFC) under cyclic electric field. Section 4 describes the characterization of the material parameters required in this formulation. Verification and prediction of constitutive model responses are also presented. Finally, Section 5 is dedicated to concluding remarks. 


\section{Formulation of Coupled Time-dependent Thermo-electro-mechanical Responses}

This section presents formulations of the constitutive and energy equations derived from TIP. For the coupled thermo-electro-mechanical responses, various dissipation effects from electromechanical stimuli occur, which results in a temperature increase and subsequent alteration of material properties. Following TIP, in a system that has energy dissipation from different external stimuli, the free energy can be expressed as a function of the observable variables (stress $\sigma_{i}$, strain $\varepsilon_{i}$, electric field $E_{i}$, electric displacement $D_{i}$ ) and other possible internal state variables (ISVs), which account for time-dependent behaviors. A linearized strain measure is considered. It is noted that Voigt notation is used for the second order tensors, i.e., the six components of strain are $\left\{\varepsilon_{1}, \varepsilon_{2}, \varepsilon_{3}, \varepsilon_{4}, \varepsilon_{5}, \varepsilon_{6}\right\}$ and similar notation is used for the corresponding stress $\left\{\sigma_{1}, \sigma_{2}, \sigma_{3}, \sigma_{4}, \sigma_{5}, \sigma_{6}\right\}$.

\subsection{Free Energy}

Following Schapery (1997) approach, we consider the following form of Gibbs free energy in terms of stresses, electric field, ISVs $\left(\xi_{m}, \xi_{m}{ }^{\prime}, \chi_{m}, \chi_{m}{ }^{\prime}\right)$ and temperature $T$, i.e.

$$
\begin{aligned}
G\left(\sigma_{i}, E_{i}, \xi_{m}, \xi_{m}^{\prime}, \chi_{m}, \chi_{m}^{\prime}, T\right)= & G_{0}-A_{m} \xi_{m}+\frac{1}{2} B_{m n} \xi_{m} \xi_{n}-P_{m} \chi_{m}+\frac{1}{2} Q_{m n} \chi_{m} \chi_{n} \\
& -C_{m} \xi_{m}^{\prime}+\frac{1}{2} D_{m n} \xi_{m}^{\prime} \xi_{n}^{\prime}-R_{m} \chi_{m}^{\prime}+\frac{1}{2} S_{m n} \chi_{m}^{\prime} \chi_{n}^{\prime}+\text { H.O.T }
\end{aligned}
$$

where $G_{0}$ is the thermo-electro-elastic Gibbs free energy, $A_{m}, P_{m}, C_{m}, R_{m}, B_{m n} Q_{m n} D_{m n}$ and $S_{m n}$ may also be a function of $\sigma_{i}, E_{i}$ and $T$. The appropriate functionality in these expressions will be explained when we derive the evolution equation for each internal state variable. In obtaining expressions for the strain and electric displacement we assume that $\boldsymbol{B}_{m n}, \boldsymbol{Q}_{m n}, \boldsymbol{D}_{m n}$ and $\boldsymbol{S}_{m n}$ are not 
a function of $\sigma_{i}$ and $E_{i}$, but can be a function of temperature, and thus the second order terms of the ISVs are dropped from the strain and electric displacement, resulting in linear relations. Using the time derivative of the Gibbs free energy and imposing the first and second laws of thermodynamics, the following constitutive relations for the strain, electric displacement and entropy per unit volume are determined from $\varepsilon_{i}=-\frac{\partial G}{\partial \sigma_{i}}, D_{i}=-\frac{\partial G}{\partial E_{i}}$ and $\eta=-\frac{\partial G}{\partial T}$, respectively, and the condition for the entropy production rate is:

$$
T \dot{\gamma} \equiv-\frac{\partial G}{\partial \xi_{m}} \dot{\xi}_{m}-\frac{\partial G}{\partial \xi_{m}^{\prime}} \dot{\xi}_{m}^{\prime}-\frac{\partial G}{\partial \chi_{m}} \dot{\chi}_{m}-\frac{\partial G}{\partial \chi_{m}^{\prime}} \dot{\chi}_{m}^{\prime}-\frac{q_{i} T_{, i}}{T} \geq 0
$$

Following Truesdell and Noll (2013) we can split the entropy production into the internal entropy production $\left(\gamma_{\text {int }}\right)$ and the entropy production from conduction $\left(\gamma_{\text {cond }}\right): T \dot{\gamma} \equiv T \dot{\gamma}_{\text {int }}+T \dot{\gamma}_{\text {cond }}$. In our study, the internal entropy production is associated with the volumetric heat generation rate $\dot{w}_{d i s}^{t} \equiv T \dot{\gamma}_{\text {int }}$ due to multiple dissipation behaviors.

The linearized time-dependent strain and electric displacement are then obtained from the following equations:

$$
\begin{gathered}
\varepsilon_{i}=-\frac{\partial G}{\partial \sigma_{i}}=-\frac{\partial G_{0}}{\partial \sigma_{i}}+\frac{\partial A_{m}}{\partial \sigma_{i}} \xi_{m}+\frac{\partial C_{m}}{\partial \sigma_{i}} \xi_{m}^{\prime} \\
D_{i}=-\frac{\partial G}{\partial E_{i}}=-\frac{\partial G_{0}}{\partial E_{i}}+\frac{\partial P_{m}}{\partial E_{i}} \chi_{m}+\frac{\partial R_{m}}{\partial E_{i}} \chi_{m}^{\prime}
\end{gathered}
$$

so that,

$$
\begin{aligned}
\varepsilon_{i}=-\frac{\partial G_{0}}{\partial \sigma_{i}}+d \varepsilon_{i} & \text { where } & d \varepsilon_{i}=\frac{\partial A_{m}}{\partial \sigma_{i}} \xi_{m}+\frac{\partial C_{m}}{\partial \sigma_{i}} \xi_{m}^{\prime} \\
D_{i}=-\frac{\partial G_{0}}{\partial E_{i}}+d D_{i} & \text { where } & d D_{i}=\frac{\partial P_{m}}{\partial E_{i}} \chi_{m}+\frac{\partial R_{m}}{\partial E_{i}} \chi_{m}^{\prime}
\end{aligned}
$$

The entropy production rate $\dot{\gamma}_{\text {int }}$ is 


$$
T \dot{\gamma}_{\mathrm{int}}=f_{m} \dot{\xi}_{m}+g_{m} \dot{\chi}_{m}+f_{m}^{\prime} \dot{\xi}_{m}^{\prime}+g_{m}^{\prime} \dot{\chi}_{m}^{\prime} \geq 0 \text { or } T \dot{\gamma}_{\mathrm{int}}=H_{1}+H_{2}+H_{3}+H_{4} \geq 0
$$

where $f_{m}=-\frac{\partial G}{\partial \xi_{m}}, g_{m}=-\frac{\partial G}{\partial \chi_{m}}, f_{m}^{\prime}=-\frac{\partial G}{\partial \xi_{m}^{\prime}}, g_{m}^{\prime}=-\frac{\partial G}{\partial \chi_{m}^{\prime}}$ and $H_{i} \quad(i=1,2,3,4)$ represents the four dissipation behaviors from the electro-mechanical conversion and their coupling effects. The entropy production rate in Eq. (7) is always positive and it is zero when $\xi_{m}=0, \xi_{m}{ }^{\prime}=0$ ,$\chi_{m}=\mathrm{O}$ and $\chi_{m}^{\prime}=0$.

There are four pairs of conjugate forces and ISVs associated with the dissipation behaviors. To determine the dissipation function of each irreversible process we need to derive the evolution equation for each ISV and their corresponding conjugate forces. In the following section we will show the derivation of these quantities for one dissipation behavior, i.e., due to mechanical effect. The rest of the dissipation functions follow a similar procedure with their respective variables. For brevity the results will be summarized in a tabular form.

\subsection{Evolution Equations for ISVs}

The expression for thermodynamic forces corresponding to the first dissipation function $\left(H_{1}\right)$ is obtained using Eq. (1) and is written as:

$$
f_{m}=-\frac{\partial G}{\partial \xi_{m}}=A_{m}-B_{m n} \xi_{n}
$$

The ISVs $\xi_{m}$ represents the time dependent deformation of piezoelectric materials, which can be associated with an arrangement of several springs and dashpots to account for time-dependent and energy dissipation effects. The ranges of $m$ and $n$ depend on the number of dashpots. To 
obtain the expression that describes the changes in the ISVs, an evolution equation that relates the thermodynamic forces and ISVs is needed, which can be written as:

$$
\dot{\xi}_{m}=c_{m n} f_{n}
$$

where $c_{m n}$ is a constant, positive definite, symmetric matrix. $f_{m}=\tilde{c}_{m n} \dot{\xi}_{n}$, where $\tilde{c}_{m n}=\left[c_{m n}\right]^{-1}$. The thermodynamic force in Eq. (8) is substituted into Eq. (9), and yields:

$$
\tilde{c}_{m n} \dot{\xi}_{n}+B_{m n} \xi_{n}=A_{m}
$$

Since $B_{m n}$ and $\tilde{c}_{m n}$ are symmetric and positive definite matrices, it is always possible to reduce them to diagonal matrices, whose scalar components are given in Eq. (10). Thus, an uncoupled set of governing equations in terms of the new principal ISVs can be written as ( $m$ not summed)

$$
\dot{\xi}_{m}+c_{m} B_{m} \xi_{m}=c_{m} A_{m}
$$

where $B_{m}$ and $c_{m}$ replace $B_{m n}$ and $c_{m n}$, respectively. The general solution of Eq. (11) is ( $m$ not summed)

$$
\xi_{m}=\int_{0}^{t} \frac{1}{B_{m}}\left[1-\exp \left[-\left(\frac{t-s}{\tau_{m}}\right)\right]\right] \frac{d A_{m}}{d s} d s
$$

where, $\tau_{m}=\left(c_{m} B_{m}\right)^{-1}$ is a positive retardation time and $t$ is the present time. It is assumed that the loading starts at initial time $t=t_{\mathrm{o}}=0$ and prior to loading (time $t<t_{\mathrm{o}}$ ) all state variables are zero, and the material is non-aging. The term $s$ is a time history, at which the input load is applied. To facilitate the characterization in terms of master creep functions through a reduce time, we assume $A_{m}=\bar{C}_{m j} \hat{\sigma}_{j}$, where $\bar{C}_{m j}$ is constant but $\hat{\sigma}_{j}$ may depend on $\sigma_{i}$ and $T$. Substituting of $A_{m}$ into Eq. (12) yields 


$$
\xi_{m}=\int_{0}^{t} \frac{\bar{C}_{m j}}{B_{m}}\left[1-\exp \left[-\left(\frac{t-s}{\tau_{m}}\right)\right]\right] \frac{d \hat{\sigma}_{j}}{d s} d s
$$

Following the similar procedure as outline above, the evolution equations for other ISVs is summarized in Table 1 and Table 2.

Table 1 Evolution of ISVs due to Mechanical Stimuli

\begin{tabular}{|c|c|c|}
\hline $\begin{array}{l}\text { Thermodynamic } \\
\text { Forces }\end{array}$ & $f_{m}=-\frac{\partial G}{\partial \xi_{m}}=A_{m}-B_{m n} \xi_{n}$ & $g_{m}^{\prime}=-\frac{\partial G}{\partial \chi_{m}^{\prime}}=R_{m}-S_{m n} \chi_{n}^{\prime}$ \\
\hline $\begin{array}{l}\text { Evolution } \\
\text { Equation }\end{array}$ & $\frac{d \xi_{m}}{d t}=c_{m n} f_{n}$ & $\frac{d \chi_{m}^{\prime}}{d t}=r_{m n}^{\prime} g_{n}^{\prime}$ \\
\hline $\begin{array}{l}\text { Constitutive } \\
\text { relation }\end{array}$ & $f_{m}=\tilde{c}_{m n} \frac{d \xi_{n}}{d t}$ & $g_{m}^{\prime}=\widetilde{r}_{m}^{\prime} \frac{d \chi_{n}^{\prime}}{d t}$ \\
\hline $\begin{array}{l}\text { Governing } \\
\text { equations }\end{array}$ & $\frac{d \xi_{m}}{d t}+c_{m} B_{m} \xi_{m}=c_{m} A_{m}$ & $\frac{d \chi_{m}^{\prime}}{d t}+r_{m}^{\prime} S_{m}^{\prime} \chi_{m}^{\prime}=r_{m}^{\prime} R_{m}$ \\
\hline $\begin{array}{l}\text { Evolution } \\
\text { equation for } \\
\text { ISVs }\end{array}$ & $\xi_{m}=\int_{0}^{t} \frac{1}{B_{m}}\left[1-\exp ^{-\left(\frac{t-s}{\tau_{m}}\right)}\right] \frac{d A_{m}}{d s} d s$ & 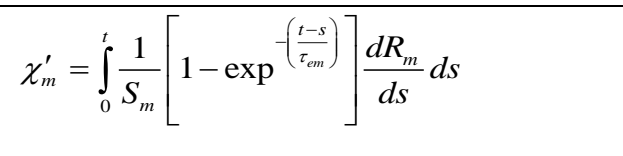 \\
\hline Retardation time & $\tau_{m}=\left(c_{m} B_{m}\right)^{-1}$ & $\tau_{e m}=\left(r_{m}^{\prime} Q_{m}^{\prime}\right)^{-1}$ \\
\hline $\begin{array}{l}\text { Gibbs free } \\
\text { energy } \\
\text { coefficients }\end{array}$ & $\begin{array}{l}A_{m}=\bar{C}_{m j} \hat{\sigma}_{j} \text {, where } \bar{C}_{m j} \text { is constant } \\
\text { but } \hat{\sigma}_{j} \text { may depend on } \sigma_{i} \text { and } T \text {. }\end{array}$ & $\begin{array}{l}R_{m}=\bar{D}_{m j}^{\prime} \tilde{\sigma}_{j}^{\prime} \text {, where } \bar{D}_{m j}^{\prime} \text { and } \bar{R}_{m j}^{\prime} \text { are } \\
\text { constants. } \tilde{\sigma}_{j}^{\prime} \text { may depend on } E_{i} \text { and } \\
T .\end{array}$ \\
\hline $\begin{array}{l}\text { Final Evolution } \\
\text { equation for } \\
\text { ISVs }\end{array}$ & $\xi_{m}=\int_{0}^{t} \frac{\bar{C}_{m j}}{B_{m}}\left[1-\exp \left[-\left(\frac{t-s}{\tau_{m}}\right)\right]\right] \frac{d \hat{\sigma}_{j}}{d s} d s$ & $\chi_{m}^{\prime}=\int_{0}^{t} \frac{\bar{D}_{m j}^{\prime}}{S_{m}}\left[1-\exp \left[-\left(\frac{t-s}{\tau_{e m}}\right)\right]\right] \frac{d \tilde{\sigma}_{j}^{\prime}}{d s} d s$ \\
\hline
\end{tabular}

Table 2 Evolution of ISVs due to Electrical Stimuli

\begin{tabular}{|l|l|l|}
\hline $\begin{array}{l}\text { Thermodyna } \\
\text { mic Forces }\end{array}$ & $f_{m}^{\prime}=-\frac{\partial G}{\partial \xi_{m}^{\prime}}=C_{m}-D_{m n} \xi_{n}^{\prime}$ & $g_{m}=-\frac{\partial G}{\partial \chi_{m}}=P_{m}-Q_{m n} \chi_{n}$ \\
\hline $\begin{array}{l}\text { Evolution } \\
\text { Equation }\end{array}$ & $\dot{\xi}_{m}^{\prime}=c_{m n}^{\prime} f_{n}^{\prime}$ & $\dot{\chi}_{m}=r_{m n} g_{n}$ \\
\hline $\begin{array}{l}\text { Constitutive } \\
\text { relation }\end{array}$ & $f_{m}^{\prime}=\tilde{c}_{m n}^{\prime} \dot{\xi}_{n}^{\prime}$ & $g_{m}=\tilde{r}_{m n} \dot{\chi}_{n}$ \\
\hline $\begin{array}{l}\text { Governing } \\
\text { differential } \\
\text { equation }\end{array}$ & $\tilde{c}_{m n}^{\prime} \dot{\xi}_{n}^{\prime}+D_{m n} \xi_{n}^{\prime}=C_{m}$ & $\tilde{r}_{m n} \dot{\chi}_{n}+Q_{m n} \chi_{n}=P_{m}$ \\
\hline
\end{tabular}




\begin{tabular}{|l|l|l|}
\hline $\begin{array}{l}\text { Evolution } \\
\text { equation for } \\
\text { ISVs }\end{array}$ & $\xi_{m}^{\prime}=\int_{0}^{t} \frac{1}{D_{m}}\left[1-\exp \left(-\frac{t-s}{\tau_{m e}}\right)\right] \frac{d C_{m}}{d s} d s$ & $\chi_{m}=\int_{0}^{t} \frac{1}{Q_{m}}\left[1-\exp -\left(\frac{t-s}{\tau_{e}}\right)\right] \frac{d P_{m}}{d s} d s$ \\
\hline $\begin{array}{l}\text { Retardation } \\
\text { time }\end{array}$ & $\tau_{m e}=\left(c_{m}^{\prime} D_{m}\right)^{-1}$ & $\tau_{e}=\left(r_{m} Q_{m}\right)^{-1}$ \\
\hline $\begin{array}{l}\text { Gibbs free } \\
\text { energy } \\
\text { coefficients }\end{array}$ & $\begin{array}{l}C_{m}=\bar{P}_{m j}^{\prime} \hat{E}_{j}^{\prime} \text {, where } \bar{P}_{m j}^{\prime} \text { is constant } \\
\text { but } \hat{E}_{j}^{\prime} \text { may depend on } \sigma_{i} \text { and } T .\end{array}$ & $\begin{array}{l}P_{m}=\bar{R}_{m j} \tilde{E}_{j}, \text { where } \bar{R}_{m j} \text { is constant but } \\
\tilde{E}_{j} \text { may depend on } E_{i} \text { and } T .\end{array}$ \\
$\begin{array}{l}\text { Evolution } \\
\text { equation for } \\
\text { ISVs }\end{array}$ & $\xi_{m}^{\prime}=\int_{0}^{t} \frac{\bar{P}_{m j}^{\prime}}{D_{m}}\left[1-\exp \left[-\left(\frac{t-s}{\tau_{m e}}\right)\right]\right] \frac{d \hat{E}_{j}^{\prime}}{d s} d s$ & $\chi_{m}=\int_{0}^{t} \frac{\bar{R}_{m j}}{Q_{m}}\left[1-\exp \left[-\left(\frac{t-s}{\tau_{e}}\right)\right]\right] \frac{d \tilde{E}_{j}}{d s} d s$ \\
\hline
\end{tabular}

\section{3 Constitutive Relations}

Using the evolution equations summarized in Table 1 and Table 2, the time-dependent part of the strain in Eq. (5) becomes

$$
\begin{aligned}
d \varepsilon_{i}=\frac{\partial A_{m}}{\partial \sigma_{i}} \xi_{m}+\frac{\partial C_{m}}{\partial \sigma_{i}} \xi_{m}^{\prime} & =\frac{\partial \hat{\sigma}_{k}}{\partial \sigma_{i}} \int_{0}^{t} \frac{\bar{C}_{m j} \bar{C}_{m k}}{B_{m}}\left[1-\exp \left[-\left(\frac{t-s}{\tau_{m}}\right)\right]\right] \frac{d \hat{\sigma}_{j}}{d s} d s \\
& +\frac{\partial \hat{E}_{k}^{\prime}}{\partial \sigma_{i}} \int_{0}^{t} \frac{\bar{P}_{m j}^{\prime} \bar{P}_{m k}^{\prime}}{D_{m}}\left[1-\exp \left[-\left(\frac{t-s}{\tau_{m e}}\right)\right]\right] \frac{d \hat{E}_{j}^{\prime}}{d s} d s
\end{aligned}
$$

The time-dependent part of the electric displacement in Eq. (6) becomes

$$
\begin{aligned}
d D_{i}=\frac{\partial P_{m}}{\partial E_{i}} \chi_{m}+\frac{\partial R_{m}}{\partial E_{i}} \chi_{m}^{\prime} & =\frac{\partial \tilde{E}_{k}}{\partial E_{i}} \int_{0}^{t} \frac{\bar{R}_{m j} \bar{R}_{m k}}{Q_{m}}\left[1-\exp \left[-\left(\frac{t-s}{\tau_{e}}\right)\right]\right] \frac{d \tilde{E}_{j}}{d s} d s \\
& +\frac{\partial \tilde{\sigma}_{k}^{\prime}}{\partial E_{i}} \int_{0}^{t} \frac{\bar{D}_{m j}^{\prime} \bar{D}_{m k}^{\prime}}{S_{m}}\left[1-\exp \left[-\left(\frac{t-s}{\tau_{e m}}\right)\right]\right] \frac{d \tilde{\sigma}_{j}^{\prime}}{d s} d s
\end{aligned}
$$

It is noted that in Eqs. (14) and (15), the lower limit of the integral is taken as 0, which indicates that the mechanical and electrical stimuli prescribed at the same time, which is at initial time $t_{0}=0$. However, the mechanical and electrical stimuli can be applied at different times, and in such situation, the lower limits in the above integrals become the times at which the stimuli are prescribed. In the above equations there are four time-dependent functions that need to be characterized from experimental data. They correspond to (i) $d D_{j k}^{M}(t)$ : deformations due to 
mechanical loading, (ii) $d P_{j k}^{\prime M E}(t)$ : deformations due to electrical input, (iii) $d P_{j k}^{E}(t)$ : electric displacements due to electrical input, and (iv) $d D_{j k}^{\prime E M}(t)$ : electric displacement due to mechanical loading. Using the above time-dependent functions, the total strain and electric displacement at present time $t$ from Eqs. (3) and (4) are expressed as

$$
\begin{aligned}
& \varepsilon_{i}(t)=-\frac{\partial G_{0}}{\partial \sigma_{i}}+\frac{\partial \hat{\sigma}_{k}}{\partial \sigma_{i}} \int_{0}^{t} d D_{j k}^{M}(t-s) \frac{d \hat{\sigma}_{j}}{d s} d s+\frac{\partial \hat{E}_{k}^{\prime}}{\partial \sigma_{i}} \int_{0}^{t} d P_{j k}^{\prime M E}(t-s) \frac{d \hat{E}_{j}^{\prime}}{d s} d s \\
& D_{i}(t)=-\frac{\partial G_{0}}{\partial E_{i}}+\frac{\partial \tilde{\sigma}_{k}^{\prime}}{\partial E_{i}} \int_{0}^{t} d D_{j k}^{\prime E M}(t-s) \frac{d \tilde{\sigma}_{j}^{\prime}}{d s} d s+\frac{\partial \tilde{E}_{k}}{\partial E_{i}} \int_{0}^{t} d P_{j k}^{E}(t-s) \frac{d \tilde{E}_{j}}{d s} d s
\end{aligned}
$$

We assume the following form of the thermo-elastic Gibbs free energy

$$
G_{0}=-\frac{1}{2} S_{i j}^{E} \sigma_{i} \sigma_{j}-\frac{1}{2} \kappa_{k j}^{\sigma} E_{j} E_{k}-d_{i k} \sigma_{i} E_{k}-\frac{1}{2} \frac{c_{\sigma}}{T_{0}} \theta^{2}-\alpha_{i} \theta \sigma_{i}-p_{i} \theta E_{i}
$$

where $S_{i j}^{E}, \kappa_{k j}^{\sigma}, d_{k i}, \alpha_{i}, p_{i}, c_{\sigma}$ are the scalar components of the elastic compliance, dielectric matrix, piezoelectric matrix, scalar components of the coefficient of thermal expansion (CTE), scalar components of the pyroelectric constants and specific heat at constant stress, respectively. $\theta=T-T_{0}$ is the temperature change with $T$ as a current temperature and $T_{0}$ is the reference temperature. The instantaneous parts of the strain and electric displacement are

$$
\varepsilon_{i}^{0}=-\frac{\partial G_{0}}{\partial \sigma_{i}}=S_{i j}^{E} \sigma_{j}+d_{i k} E_{k}+\alpha_{i} \theta ; \quad D_{i}^{0}=-\frac{\partial G_{0}}{\partial E_{i}}=d_{i k} \sigma_{k}+\kappa_{i j}^{\sigma} E_{j}+p_{i} \theta
$$

Using Eq. (19) and after some simplifications, for a linear electro-mechanical behavior, the timedependent strain and electric displacements in Eqs. (16) and (17) are now written as:

$$
\begin{gathered}
\varepsilon_{i}(t)=S_{i j}^{E} \sigma_{j}(t)+d_{i k} E_{k}(t)+\int_{0}^{t} \Delta S_{i j}^{E}(t-s) \frac{d \sigma_{j}}{d s} d s+\int_{0}^{t} \Delta d_{i j}^{M E}(t-s) \frac{d E_{j}}{d s} d s+\alpha_{i} \theta \\
D_{i}(t)=d_{i k} \sigma_{k}(t)+\kappa_{i j}^{\sigma} E_{j}(t)+\int_{0}^{t} \Delta d_{i j}^{E M}(t-s) \frac{d \sigma_{j}}{d s} d s+\int_{0}^{t} \Delta \kappa_{i j}^{\sigma}(t-s) \frac{d E_{j}}{d s} d s+p_{i} \theta
\end{gathered}
$$


where $\Delta S_{i j}^{E}(t), \Delta d_{i j}^{M E}(t), \Delta d_{i j}^{E M}(t)$ and $\Delta \kappa_{i j}^{\sigma}(t)$ are the time dependent kernel functions corresponding to the elastic, electromechanical coupling due to electrical input, electromechanical coupling due to mechanical loading and permittivity tensor, respectively. The strain $\varepsilon_{i}$ and electric displacement $D_{i}$ are evaluated at current time $t$ and the input history is prescribed at $0<s<t$. As mentioned above in case the mechanical and electrical inputs are prescribed at different times, the lower limits of the integrals in Eqs. (20) and (21) will correspond to the times at which the stimuli are prescribed. We use Prony series to represent the time dependent kernels in piezoelectric materials:

$$
\begin{array}{ll}
\Delta S_{i j}^{E}(t)=\sum_{I=1}^{N P}{ }^{I} S_{i j}^{E}\left(1-\exp \left[-{ }^{I} \lambda_{i j}^{E} t\right]\right) ; & \Delta d_{i j}^{M E}(t)=\sum_{I=1}^{N P}{ }^{I} d_{i j}^{M E}\left(1-\exp \left[-{ }^{I} \lambda_{i j}^{M E} t\right]\right) \\
\Delta \kappa_{i j}^{\sigma}(t)=\sum_{I=1}^{N P}{ }^{I} \kappa_{i j}^{\sigma}\left(1-\exp \left[-{ }^{I} \lambda_{i j}^{\sigma} t\right]\right) ; & \Delta d_{i j}^{E M}(t)=\sum_{I=1}^{N P}{ }^{I} d_{i j}^{E M}\left(1-\exp \left[-{ }^{I} \lambda_{i j}^{E M} t\right]\right)
\end{array}
$$

Where ${ }^{I} S_{i j}^{E},{ }^{I} d_{i j}^{M E},{ }^{I} d_{i j}^{E M},{ }^{I} \kappa^{\sigma}{ }_{i j},{ }^{I} \lambda_{i j}^{E},{ }^{I} \lambda_{i j}^{M E},{ }^{I} \lambda_{i j}^{E M}$ and ${ }^{I} \lambda_{i j}^{\sigma}$ are the material parameters related to $I^{\text {th }}$ component of Prony series. The above equations are the time dependent functions corresponding to the coupled electro-mechanical response of piezoelectric materials. The material parameters corresponding to these time-dependent functions are usually determined from creep, relaxation and hysteresis type experimental data under electrical or mechanical stimulus.

\section{4. Energy Equation}

Combining the first and second law of thermodynamics ((Rajagopal and Srinivasa, 2011), (Khan and Muliana, 2012)), the following expression for the energy balance in electro-thermoviscoelastic materials can be established:

$$
-T \frac{d}{d t}\left\{\frac{\partial G}{\partial T}\right\}=-q_{i, i}+T \dot{\gamma}_{\mathrm{int}}
$$


The rate of internal entropy generation, $T \dot{\gamma}_{\text {int }}$, given in Eq. (7) can be found using the conjugate forces and evolution equation of each ISV developed in previous section. For the linear time-dependent electro-mechanical responses, the components of the total heat generation due to mechanical stimuli are expressed as

$$
\begin{aligned}
& H_{1}=f_{m} \dot{\xi}_{m}=\left[\sigma_{k}-\int_{0}^{t}\left[1-\exp ^{-\left(\frac{t-s}{\tau_{m}}\right)}\right] \frac{d \sigma_{k}}{d s} d s\right] \frac{d}{d t}\left(\Delta S_{j k}^{E}(t-s) \frac{d \sigma_{j}}{d s} d s\right) \\
& H_{4}=g_{m}^{\prime} \dot{\chi}_{m}^{\prime}=\left[\sigma_{k}-\int_{0}^{t}\left[1-\exp ^{-\left(\frac{t-s}{\tau_{e m}}\right)}\right] \frac{d \sigma_{k}}{d s} d s\right] \frac{d}{d t}\left(\int_{0}^{t} \Delta d_{j k}^{E M}(t-s) \frac{d \sigma_{j}}{d s} d s\right)
\end{aligned}
$$

while the components of the heat generation due to electrical stimuli are

$$
\begin{aligned}
& H_{2}=g_{m} \dot{\chi}_{m}=\left[E_{k}-\int_{0}^{t}\left[1-\exp ^{-\left(\frac{t-s}{\tau_{e}}\right)}\right] \frac{d E_{k}}{d s} d s\right] \frac{d}{d t}\left(\int_{0}^{t} \Delta \kappa_{i j}^{\sigma}(t-s) \frac{d E_{j}}{d s} d s\right) \\
& H_{3}=f_{m}^{\prime} \dot{\xi}_{m}^{\prime}=\left[E_{k}-\int_{0}^{t}\left[1-\exp ^{-\left(\frac{t-s}{\tau_{m e}}\right)}\right] \frac{d E_{k}}{d s} d s\right] \frac{d}{d t}\left(\int_{0}^{t} \Delta d_{j k}^{M E}(t-s) \frac{d E_{j}}{d s} d s\right)
\end{aligned}
$$

Using Eq. (1) and Eq. (18), the rate of entropy generation can be expressed as

$$
-T \frac{d}{d t}\left\{\frac{\partial G}{\partial T}\right\}=\rho c_{\sigma}(T) \dot{T}+T \alpha_{i} \dot{\sigma}_{i}+T p_{i} \dot{E}_{i}
$$

For a linear electro-mechanical behavior and temperature dependent material properties, the energy equation becomes 


$$
\begin{aligned}
& \rho(T) c_{\sigma}(T) \dot{T}+T \alpha_{i}(T) \dot{\sigma}_{i}+T p_{i}(T) E_{i}=-q_{i, i} \\
& {\left[\left\{\sigma_{k}-\int_{0}^{t}\left[1-\exp \left[-\left(\frac{t-s}{\tau_{m}}\right)\right]\right] \frac{d}{d s}\left(\sigma_{k}\right) d s\right\} \frac{d}{d t}\left(\Delta S_{j k}^{E}(t-s, T) \frac{d}{d s}\left(\sigma_{j}\right) d s\right)\right.} \\
& +\left[\left\{E_{k}-\int_{0}^{t}\left[1-\exp \left[-\left(\frac{t-s}{\tau_{e}}\right)\right] \frac{d}{d s}\left(E_{k}\right) d s\right\}\right] \frac{d}{d t}\left(\int_{0}^{t} \Delta \kappa_{i j}^{\sigma}(t-s, T) \frac{d}{d s}\left(E_{j}\right) d s\right)\right. \\
& +\left[\left\{E_{k}-\int_{0}^{t}\left[1-\exp \left[-\left(\frac{t-s}{\tau_{m e}}\right)\right]\right] \frac{d}{d s}\left(E_{k}\right) d s\right\}\right] \frac{d}{d t}\left(\int_{0}^{t} \Delta d_{j k}^{M E}(t-s, T) \frac{d}{d s}\left(E_{j}\right) d s\right) \\
& +\left[\left\{\sigma_{k}-\int_{0}^{t}\left[1-\exp \left[-\left(\frac{t-s}{\tau_{e m}}\right)\right]\right] \frac{d}{d s}\left(\sigma_{k}\right) d \Omega^{\prime \prime \prime}\right\}\right] \frac{d}{d t}\left(\int_{0}^{t} \Delta d_{j k}^{E M}(t-s, T) \frac{d}{d s}\left(\sigma_{j}\right) d s\right)
\end{aligned}
$$

and the constitutive relations can be expressed as

$$
\begin{aligned}
& \varepsilon_{i}(t, T)=S_{i j}^{E}(T) \sigma_{j}(t)+d_{i k}(T) E_{k}(t)+\int_{0}^{t} \Delta S_{i j}^{E}(t-s, T) \frac{d \sigma_{j}}{d s} d s \\
& +\int_{0}^{t} \Delta d_{i j}^{M E}(t-s, T) \frac{d E_{j}}{d s} d s+\alpha_{i}(T) \theta \\
& D_{i}(t, T)=d_{i k}(\mathrm{~T}) \sigma_{k}(t)+\kappa_{i j}^{\sigma}(T) E_{j}(t)+\int_{0}^{t} \Delta d_{i j}^{E M}(t-s, T) \frac{d \sigma_{j}}{d s} d s \\
& +\int_{0}^{t} \Delta \kappa_{i j}^{\sigma}(t-s, T) \frac{d E_{j}}{d s} d s+p_{i}(T) \theta
\end{aligned}
$$

\section{Experimental Characterization}

This section discusses experimental characterization of PZT fibers and active fiber composites (AFC) under cyclic electric field inputs at different frequencies and amplitudes. The frequencies are chosen in order to avoid resonance regime and Joule heating. The PZT5A fibers and AFCs were purchased from Advanced Cerametrics, Inc. AFCs are long fiber composites in which the fibers are made from PZT. A schematic of an AFC is shown in Figure 1. The fibers are the same PZT fibers used to manufacture AFCs and have a diameter of $0.25 \mathrm{~mm}$; they are embedded in an epoxy matrix to give the composite more flexibility and ductility. The AFCs are $120 \mathrm{~mm}$ long, $24 \mathrm{~mm}$ wide, and $0.38 \mathrm{~mm}$ thick. As shown, the AFC utilizes interdigitated 
electrodes (IDEs) along the direction of the fibers (Bent and Hagood, 1995). The small electrode gap in the IDEs makes it possible to apply a high electric field through the fibers without applying high voltage.
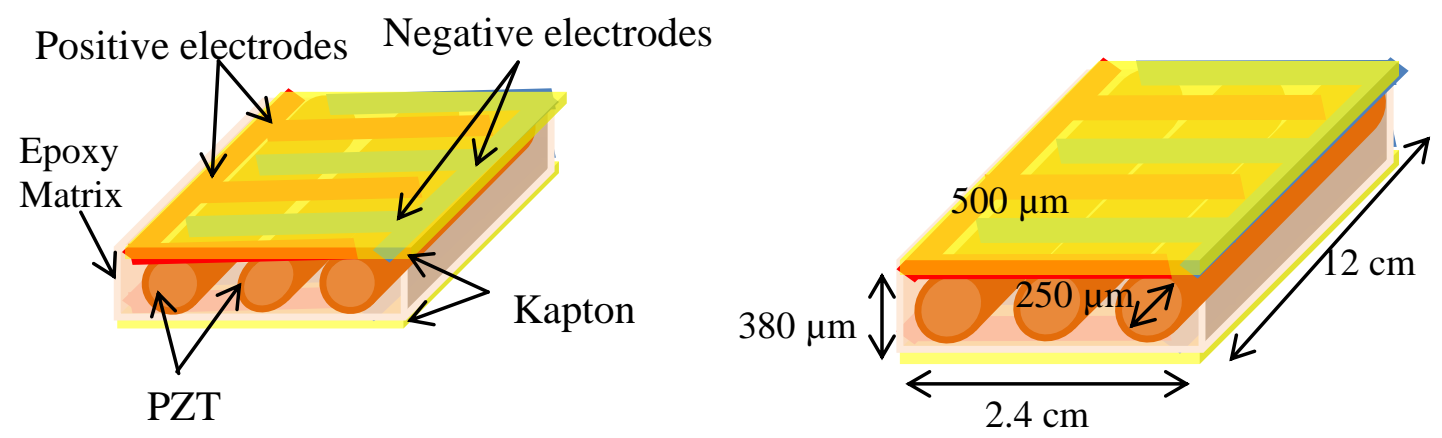

Figure 1 Schematic of AFCs: a) constituents and b) typical dimensions

Several measurement setups are used for the electrical and electro-mechanical measurements of PZT5A fibers and AFC. The electrical measurements of AFCs were completed in a silicone oil bath as a dielectric medium to avoid shorting and arcing due to the high electric field. The sample was connected to a voltage generator through a high voltage power amplifier. The amplifier is equipped with a voltage and a current monitors that were connected to an oscilloscope to measure the electric current and the voltage. Data were collected on a laptop using GPIB cable and Labview program and the current was integrated with respect to time to obtain the electric displacement which can be interchangeable with polarization for AFC and PZT. In AFCs, the electric current is passed through interdigitated electrodes, in which the electric field is not uniformly distributed in the fibers and the charges are collected on the top and bottom of AFCs by the electrode fingers (see Figure 1). In order to determine electric displacement and polarization during the measurement, the collected charges are divided by the surface area, which for the AFCs is the sum of the cross section of the AFC under the electrodes $(0.25 \times 20 \mathrm{~mm})$ multiplied by the number of electrode gaps (170). For strain measurement, the 
displacement in the sample was measured in ambient air using a high-resolution fiber optic sensor (MTI 2100) and the displacement and voltage data were measured using an oscilloscope, then saved digitally.

In order to perform experiments on single fibers, two methods were used. The first method consisted of printing IDE electrodes on Kapton sheets and using them to apply the electric field and collect charges as shown in Figure 2. PZT fibers were placed between two sheets of the IDE/Kapton and a vacuum was applied to ensure contact between the fibers and the electrodes. The electric displacement was then determined similarly to the AFC measurement, discussed above. This set up was used to simulate the non-uniform electric field applied on the PZT fibers when embedded in AFCs. The second method consisted of testing a single fiber of a length of $2.75 \mathrm{~mm}$, as in the setup shown in Figure 3. The short fiber is placed between two semi-spherical electrodes where a uniform electric field is applied on the fiber. The entire setup is submersed in Galden oil. The setup is connected to a Sawyer-Tower circuit to measure polarization and an LVDT to measure the displacement generated by the fibers. The PZT fibers used here are sintered at high temperature $(>800 \mathrm{oC})$ so they are not flexible green fibers; they are brittle and stiff enough to stand without bending or folding. Experimental tests were first done under high electric field in order to determine the coercive electric field limits. Table 3 summarizes the ferroelectric properties for AFCs and fibers for different experimental setups. When the properties of PZT fibers from different setups and those of AFCs are compared, it is found that the remnant and saturation polarization of AFCs and those of the PZT fiber with IDE electrodes are similar and much smaller than the values for the PZT fiber with parallel electrodes. When the IDE electrodes are used, the local electric field inside the fibers are different in magnitude and direction from the applied electric field. The non-uniform electric field in the fibers also result in 
higher coercive field in the AFC and PZT fiber with IDE electrodes. In this study, the magnitudes of electric fields considered are lower than the coercive electric field of the materials.

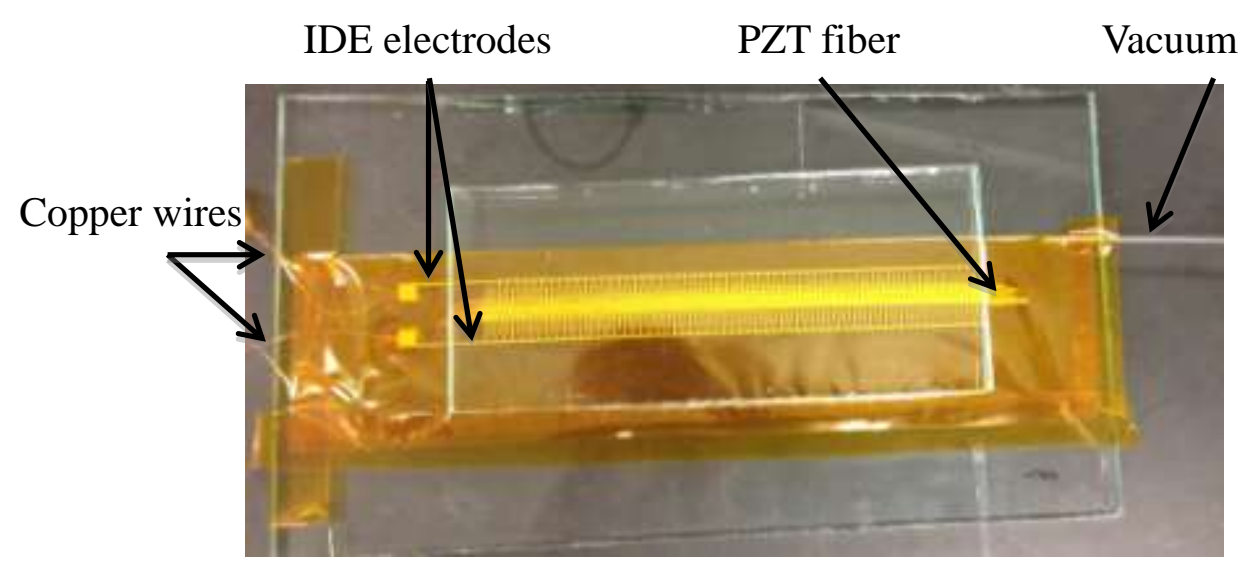

Figure 2 IDE experimental setup for electrical characterization of PZT fibers

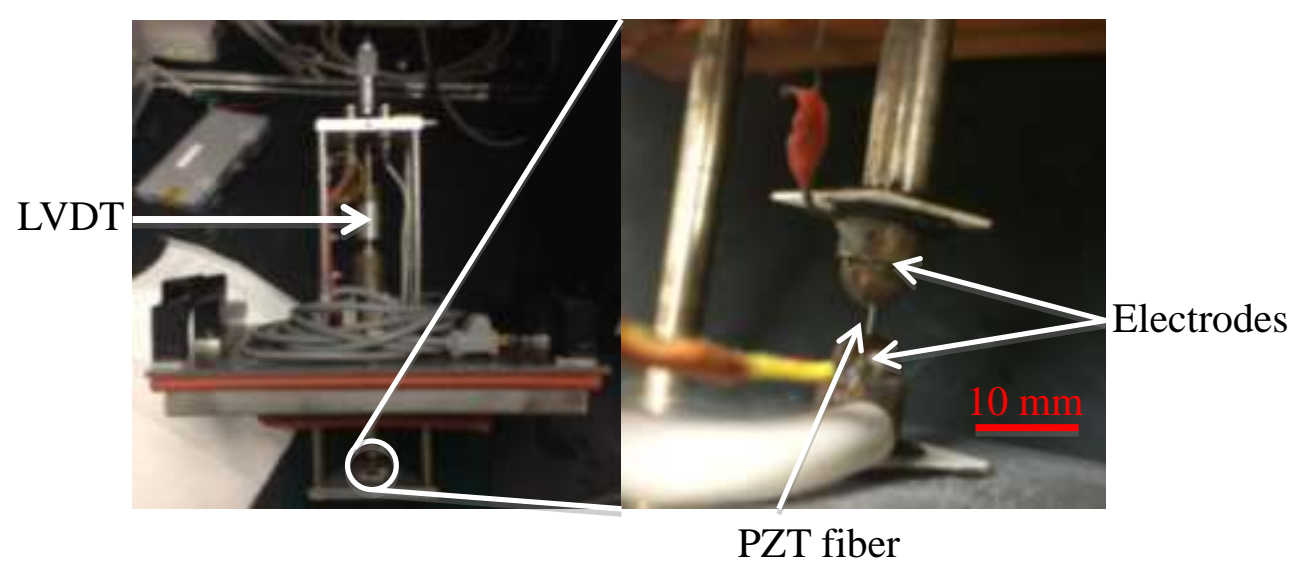

Figure 3 Parallel electrodes setup for electrical characterization of PZT fibers

Table 3 Comparison of ferroelectric properties for different experimental setups

\begin{tabular}{|l|l|l|l|l|}
\hline Properties & $P_{\mathrm{s}}\left(\mathrm{C} / \mathrm{m}^{2}\right)$ & $P_{\mathrm{r}}\left(\mathrm{C} / \mathrm{m}^{2}\right)$ & $E_{\mathrm{c}}(\mathrm{MV} / \mathrm{m})$ & $E_{\max }(\mathrm{MV} / \mathrm{m})$ \\
\hline AFC & 0.14 & 0.11 & 1.4 & 2.5 \\
PZT fiber (IDE) & 0.14 & 0.11 & 1.7 & 2.1 \\
PZT fiber (parallel electrodes) & 0.34 & 0.21 & 1.02 & 2.5 \\
\hline
\end{tabular}




\section{Coupled thermo-electro-mechanical responses of PZTs and AFC}

In this section, we simulate coupled thermo-electro-mechanical responses of soft PZTs subjected to various loading histories. We studied the strain, polarization and heat generation behaviors in PZT and AFC due to cyclic electric field at different amplitude and frequencies. Experimental data from (Zhou and Kamlah, 2006) on soft PZT are used to further compare with the properties of PZT fiber. Parametric studies on the effect of temperature increases on the overall hysteretic responses are also presented.

\subsection{Calibration of Material Parameters}

Most of experimental tests in literature are performed under uniaxial electric field and/or compressive stress loading, which reduce the numbers of nonzero components of the field variables. It is often assumed that the uniaxial external load is applied in the $x_{3}$ direction, which is also the poling direction of a polarized specimen. Then, the strain and electric displacement along the poling direction, ignoring the thermal expansion and pyroelectric effect, is expressed as

$$
\begin{gathered}
\varepsilon_{3}(t)=S_{33}^{E} \sigma_{3}(t)+d_{33} E_{3}(t)+\int_{0}^{t} \Delta S_{33}^{E}(t-s) \frac{d}{d s}\left(\sigma_{3}\right) d s+\int_{0}^{t} \Delta d_{33}^{M E}(t-s) \frac{d}{d s}\left(E_{3}\right) d s \\
D_{3}(t)=d_{33} \sigma_{3}(t)+\kappa_{33}^{\sigma} E_{3}(t)+\int_{0}^{t} \Delta d_{33}^{E M}(t-s) \frac{d}{d s}\left(\sigma_{3}\right) d s+\int_{0}^{t} \Delta \kappa_{33}^{\sigma}(t-s) \frac{d}{d s}\left(E_{3}\right) d s
\end{gathered}
$$

In the direction perpendicular to the poling, the transverse strains are given by

$$
\varepsilon_{1}(t)=\varepsilon_{2}(t)=S_{13}^{E} \sigma_{3}(t)+d_{31} E_{3}(t)+\int_{0}^{t} \Delta S_{13}^{E}(t-s) \frac{d}{d s}\left(\sigma_{3}\right) d s+\int_{0}^{t} \Delta d_{31}^{M E}(t-s) \frac{d}{d s}\left(E_{3}\right) d s
$$

The rest of strain and dielectric components are zero. The above equations are used to describe the coupled response of piezoelectric materials. A recursive iterative numerical method developed by (Muliana and Khan, 2008) is used to solve Eq. (30)-(32). The time dependent functions are easily calibrated by applying the external stimuli independently. For example, if an 
electric field input is applied $\left(\sigma_{3}=0\right)$ then the components that involve stress $\sigma_{3}$ in the constitutive relations in Eqs. (30)-(32) drop to zero, and the following time-dependent material parameters can be determined $d_{33}^{M E}(t), d_{31}^{M E}(t)=d_{32}^{M E}(t), \quad{ }_{3}(t)$. The energy equation in Eq. (27) is further simplified as

$$
\begin{aligned}
& \rho c_{\sigma} \dot{T}= \\
& +\left[\left\{E_{3}-\int_{0}^{t}\left[1-\exp \left[-\left(\frac{t-s}{\tau_{e}}\right)\right]\right] \frac{d}{d s}\left(E_{3}\right) d s\right\}\right] \frac{d}{d t}\left(\int_{0}^{t} \Delta \kappa_{33}^{\sigma}(t-s) \frac{d}{d s}\left(E_{3}\right) d s\right) \\
& +\left[\left\{E_{3}-\int_{0}^{t}\left[1-\exp \left[-\left(\frac{t-s}{\tau_{m e}}\right)\right]\right] \frac{d}{d s}\left(E_{3}\right) d s\right\}\right] \frac{d}{d t}\left(\int_{0}^{t} \Delta d_{33}^{M E}(t-s) \frac{d}{d s}\left(E_{3}\right) d s\right) \\
& +\left[\left\{E_{3}-\int_{0}^{t}\left[1-\exp \left[-\left(\frac{t-s}{\tau_{m e}}\right)\right]\right] \frac{d}{d s}\left(E_{3}\right) d s\right\}\right] \frac{d}{d t}\left(\int_{0}^{t} \Delta d_{31}^{M E}(t-s) \frac{d}{d s}\left(E_{3}\right) d s\right) \\
& +\left[\left\{E_{3}-\int_{0}^{t}\left[1-\exp \left[-\left(\frac{t-s}{\tau_{m e}}\right)\right]\right] \frac{d}{d s}\left(E_{3}\right) d s\right\}\right] \frac{d}{d t}\left(\int_{0}^{t} \Delta d_{32}^{M E}(t-s) \frac{d}{d s}\left(E_{3}\right) d s\right)
\end{aligned}
$$

Based on documented experimental evidence of the temperature-dependent responses of piezoelectric ceramics ((Ben Atitallah et al., 2010)), the relations in Eqs. (28)-(29) and energy equation Eq. (27) should be used to predict the responses.

\subsubsection{Responses under constant electric field}

Zhou and Kamlah (2006) showed that both electric displacement and strain in the bulk PIC151 soft PZT increases with time when a constant electric field is applied. In such cases, the time dependent electric permittivity and piezoelectric constants can be characterized by using Eqs. (30)-(32). Figure 4 shows the experimental data and calibrated response of time dependent

dielectric permittivity determined from the creep polarization $\left(\mathrm{C} / \mathrm{m}^{2}\right)$ and time dependent piezoelectric coefficient obtained from the creep strain response under constant electric field of 
$0.5 \mathrm{MV} / \mathrm{m}$. Table 4 shows the calibrated instantaneous (time-independent) material properties and Prony series coefficients from the experimental data for the time dependent kernel functions given in Eqs. (30) and (32). The specific heat and density of the PIC151 soft PZT are taken from (Zhou and Kamlah (2006)). The experimental conditions and loading rates are different from the manufacturer therefore all the instantaneous coupling coefficients and dielectric properties are not taken from the manufacturer data sheet.
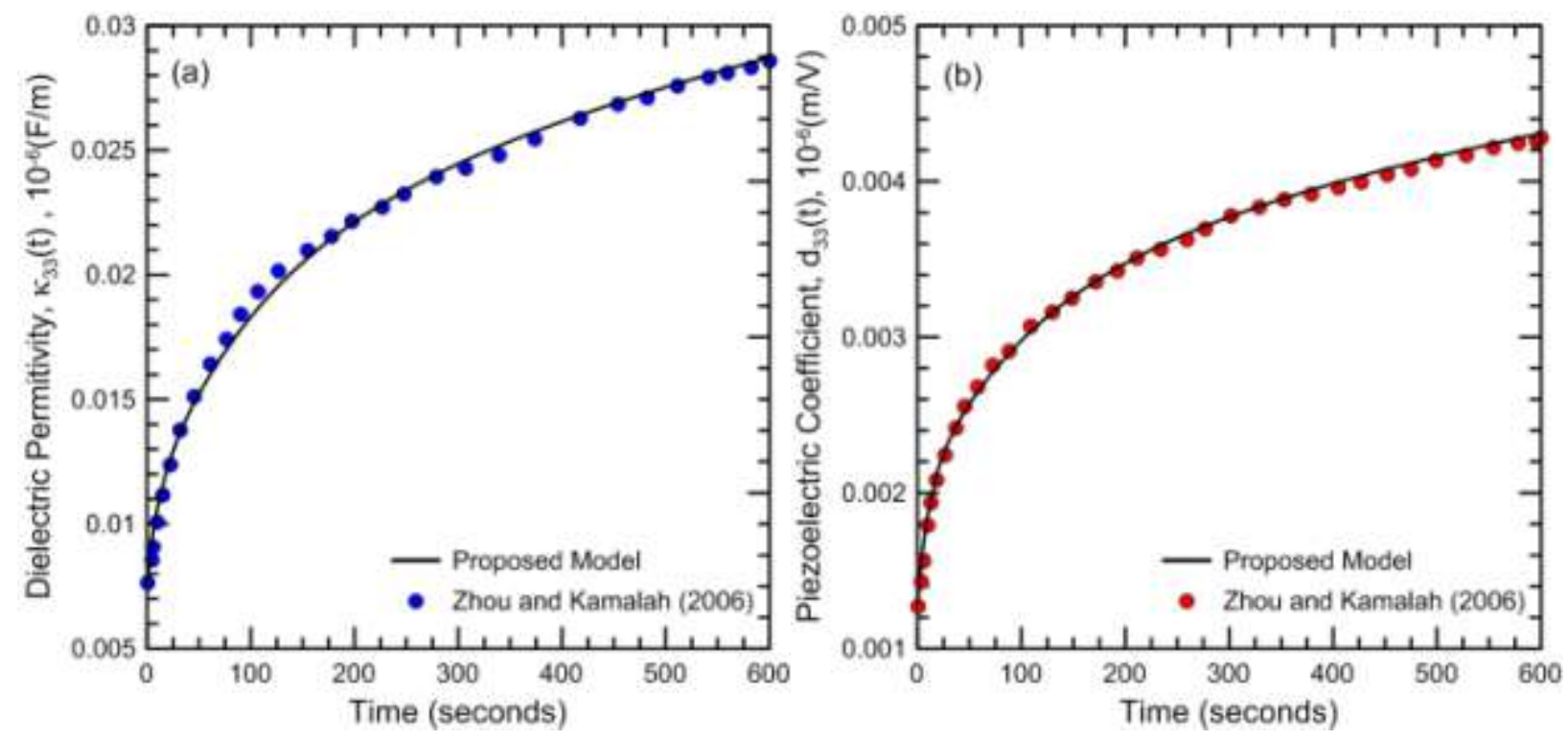

Figure 4 Calibrated and Experimental curve for time dependent (a) Dielectric Permittivity (b) Piezoelectric coefficients at constant electric field (E3) of 0.5MV/m.

Table 4 Prony series coefficients, mechanical, electromechanical and physical properties of PIC151 soft PZT.

\begin{tabular}{|c|c|c|c|c|}
\hline $\mathrm{NP}$ & ${ }^{I} \lambda_{33}^{M E}\left(\sec ^{-1}.\right)$ & ${ }^{I} d_{33}^{M E}\left(10^{-12} \mathrm{~m} / \mathrm{V}\right)$ & ${ }^{I} \lambda_{33}^{\sigma}\left(\sec ^{-1}.\right)$ & ${ }^{I} \boldsymbol{\kappa}_{33}^{\sigma}\left(10^{-9} \mathrm{~F} / \mathrm{m}\right)$ \\
\hline 1 & 1 & 10 & 1 & 100 \\
\hline 2 & 0.1 & 700 & 0.1 & 3000 \\
\hline 3 & 0.01 & 1200 & 0.01 & 9000 \\
\hline 4 & 0.001 & 2500 & 0.001 & 20000 \\
\hline \multicolumn{5}{|c|}{ Specific heat, $(c)=420 . \mathrm{J} / \mathrm{kg}^{\circ} \mathrm{K}$} \\
\hline \multicolumn{5}{|c|}{ Density, $(\rho)=7800 \mathrm{Kg} / \mathrm{m}^{3}$} \\
\hline \multicolumn{5}{|c|}{ Time independent Material Properties } \\
\hline \multicolumn{5}{|c|}{ Piezoelectric Charge Constants: $d_{33}^{M E}=1270 \times 10^{-12} \mathrm{~m} / \mathrm{V}$. } \\
\hline \multicolumn{5}{|c|}{ Relative Permittivity Constants: $\kappa_{33}^{\sigma} / \kappa_{0}=860$} \\
\hline \multicolumn{5}{|c|}{ Dielectric Permittivity of vacuum: $\kappa_{0}=8.854 \times 10^{-12} \mathrm{~F} / \mathrm{m}$} \\
\hline
\end{tabular}




\subsubsection{Responses under cyclic electric field}

\section{a) PZT fiber (Interdigitated electrodes setup)}

Experiments on PZT 5A fibers and AFCs under cyclic electric fields have been conducted as discussed above. For the material parameter calibrations in this study, we consider experimental results under linear hysteretic responses with amplitude of electric field below the coercive electric field of the materials. The coercive electric field of PZT 5A fiber measured using IDE method was determined to be $E_{\mathrm{c}}=1.7 \mathrm{MV} / \mathrm{m}$. Figure 5 shows the experimental data and calibrated response of hysteretic polarization under voltage amplitude of $800 \mathrm{~V}\left(E_{3}=1.19 \mathrm{MV} / \mathrm{m}\right)$ and frequency $1 \mathrm{~Hz}$. Table 5 shows the calibrated instantaneous material properties and Prony series coefficients from the experimental data for the time dependent kernel functions given in Eq. (32). The specific heat and density of the PZT 5A fibers are taken from the manufacturer data sheet (Advanced Cerametrics Inc.), and the rest of data are calibrated from experiment. Predictions of hysteretic polarization responses of PZT fibers under different amplitude of electric field are shown in Figure 6. The model, within a linear time-dependent electromechanical responses, can capture the experimental responses very well at $\mathrm{E}=1.34 \mathrm{MV} / \mathrm{m}$. However, as electric field increases, some deviations are observed when the hysteresis curve tends to change its shapes from ellipsoidal to non-ellipsoidal, which cannot be captured well by the proposed model. 


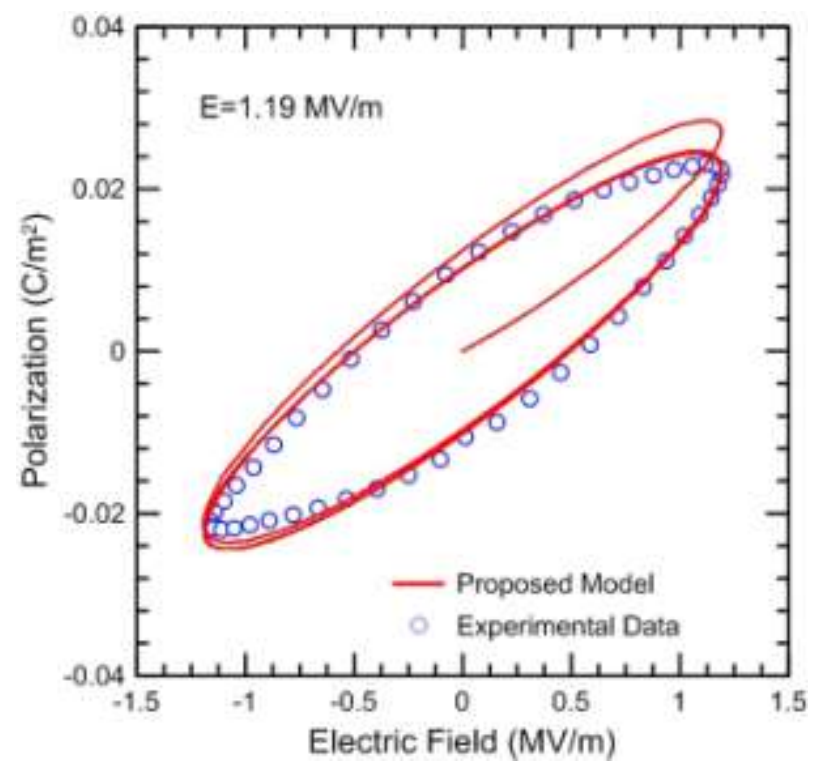

Figure 5 Calibrated and experimental curve for time dependent hysteresis (electric field vs polarization) behavior of soft PZT fiber 5A.
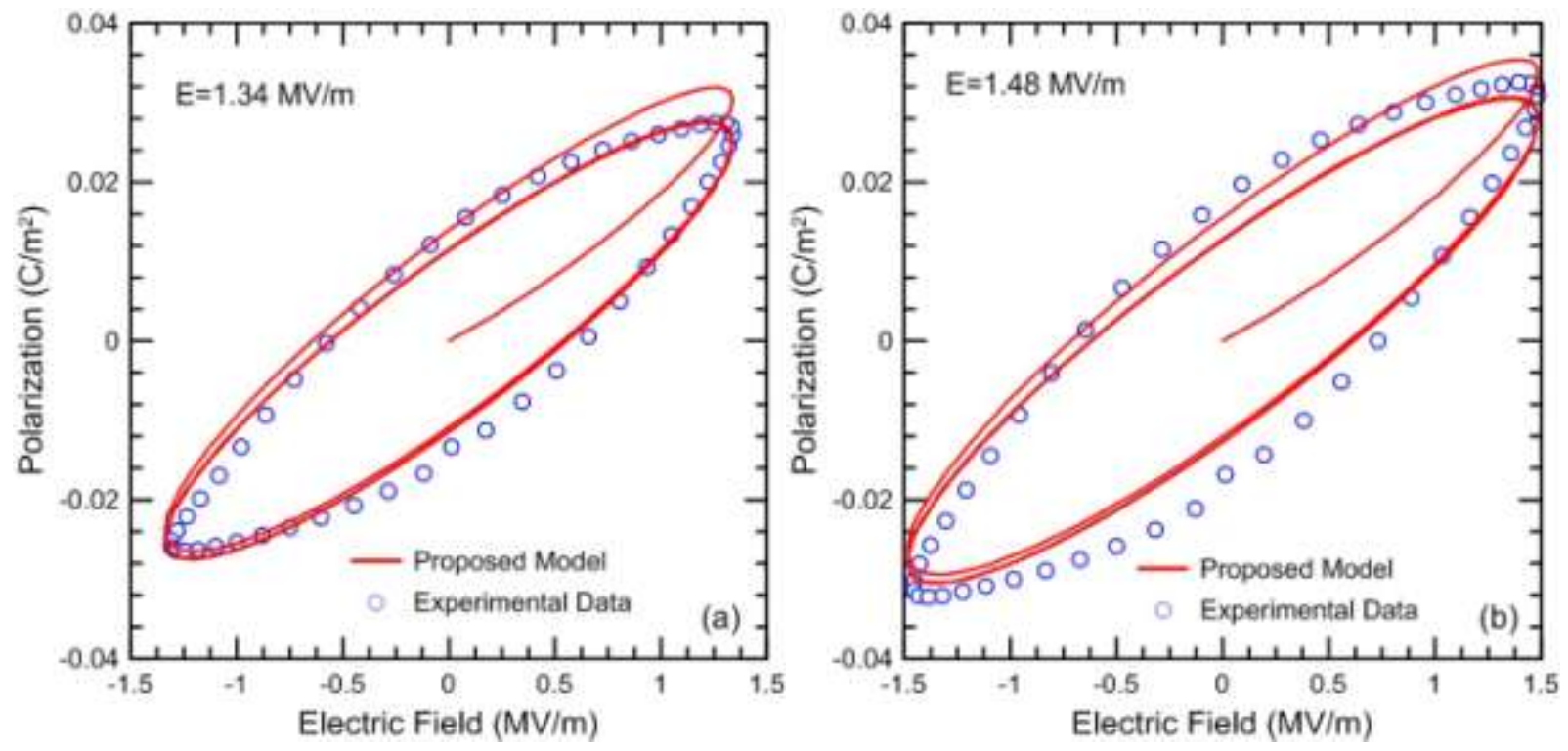

Figure 6 Predicted and experimental curve for time dependent hysteresis (electric field vs polarization) behavior of soft PZT fiber $5 \mathrm{~A}$ at (a) $\mathrm{E}=1.34$ and (b) $\mathrm{E}=1.48 \mathrm{MV} / \mathrm{m}$.

Table 5 Prony series coefficients, mechanical, electromechanical and physical properties of PZT 5A fiber.

\begin{tabular}{|l|l|l|l|}
\hline $\mathrm{NP}$ & ${ }^{I} \lambda_{33}^{\sigma}\left(\mathrm{sec}^{-1}.\right)$ & ${ }^{I} \kappa^{\sigma}{ }_{33}\left(10^{-9} \mathrm{~F} / \mathrm{m}\right)$ & $\begin{array}{l}\text { Specific heat, }(c)=420 . \mathrm{J} / \mathrm{kg}{ }^{\circ} \mathrm{K} \\
\text { Density, }(\rho)=7800 \mathrm{Kg} / \mathrm{m}^{3}\end{array}$ \\
\hline 1 & 3.0 & 22 & Den \\
\hline
\end{tabular}


Time independent Material Properties

Piezoelectric Charge Constants: $d_{33}^{M E}=400 \times 10^{-12} \mathrm{~m} / \mathrm{V}$.

Relative Permittivity Constants: $\kappa_{33}^{\sigma} / \kappa_{0}=1750$

\section{b) PZT fiber (with parallel electrode)}

We also calibrated the material parameters for the PZT fibers measured using the parallel electrodes setup. The polarization and strain hysteretic responses under several amplitude of electric field ranging from 0.2-0.8 MV/m and at different frequencies, i.e., $0.2,1.0$ and $5 \mathrm{~Hz}$ were considered. Figure 7(a)-(b) shows the experimental data and calibrated response of hysteretic polarization and strain under electric field of $E_{3}=0.3 \mathrm{MV} / \mathrm{m}$ ) and at a frequency of $5 \mathrm{~Hz}$. Table 6 shows the corresponding Prony series coefficients for the time dependent kernel function given in Eqs. (30) and (32). The instantaneous (time-independent) material properties and other physical properties are also given for the PZT 5A fibers.

Table 6 Prony series coefficients, mechanical, electromechanical and physical properties of PZT fiber with parallel electrode setup.

\begin{tabular}{|l|l|l|l|l|}
\hline $\mathrm{NP}$ & ${ }^{I} \lambda_{33}^{M E}\left(\mathrm{sec}^{-1}.\right)$ & ${ }^{I} d_{33}^{M E}\left(10^{-12} \mathrm{~m} / \mathrm{V}\right)$ & ${ }^{I} \lambda_{33}^{\sigma}\left(\mathrm{sec}^{-1}.\right)$ & ${ }^{I} \kappa_{33}^{\sigma}\left(10^{-9} \mathrm{~F} / \mathrm{m}\right)$ \\
\hline 1 & 0.5 & 100 & 0.5 & 10 \\
\hline 2 & 1.0 & 100 & 1.5 & 10 \\
\hline 3 & 2.0 & 300 & 3.0 & 10 \\
\hline 4 & 50.0 & 500 & 50.0 & 11 \\
\hline Specific heat, $(c)=420 . \mathrm{J} / \mathrm{kg}{ }^{\circ} \mathrm{K}$ \\
Density, $(\rho)=7800 \mathrm{Kg} / \mathrm{m}^{3}$ \\
Time independent Material Properties \\
Piezoelectric Charge Constants: $\quad d_{33}^{M E}=110 \times 10^{-12} \mathrm{~m} / \mathrm{V}$. \\
Relative Permittivity Constants: $\kappa_{33}^{\sigma} / \kappa_{0}=4700$ \\
\hline
\end{tabular}



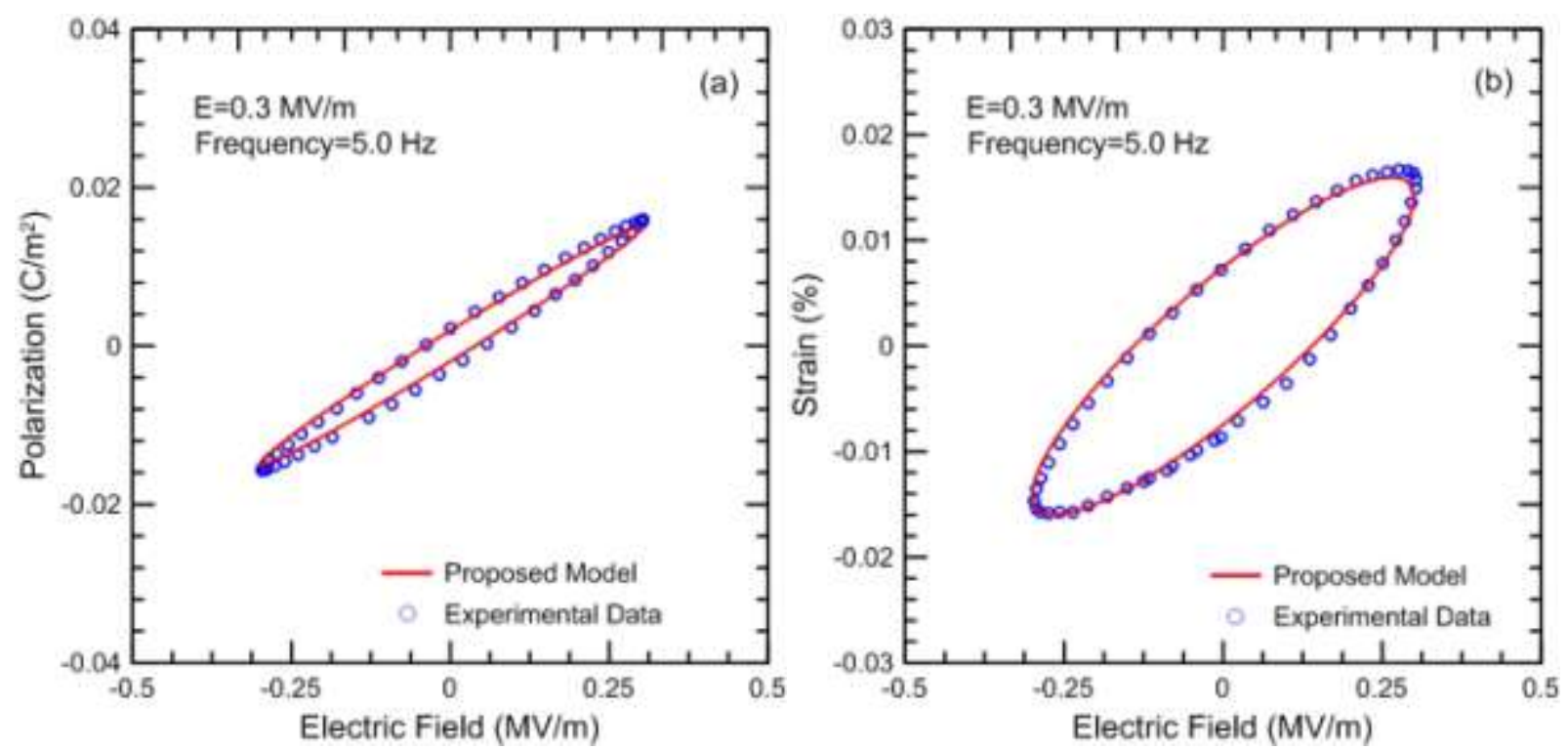

Figure 7 Calibrated and experimental curve for time dependent hysteresis a) electric field vs polarization b) electric field vs strain behavior of PZT fiber at electric field (E3) of $0.3 \mathrm{MV} / \mathrm{m}$ and frequency of $\mathrm{f}=5.0 \mathrm{~Hz}$.

Next we predict the hysteretic responses of PZT fiber obtained from the parallel electrode setup that were not used in the calibration process. The responses of PZT fibers at different amplitudes and at frequency of $5 \mathrm{~Hz}$ are shown in Figure 8 (a)-(f). Pretty good predictions are obtained for the hysteretic strains at all electric field amplitudes. However for polarization at higher magnitudes, the results start to deviate and we attribute this mismatch to unaccounted nonlinear behavior. The hysteretic responses of PZT fiber at different amplitudes and at different frequencies other than the ones used for calibration are also compared. Figure 9 (a)-(f) shows the polarization and strain hysteretic responses at 0.2 and $0.4 \mathrm{MV} / \mathrm{m}$ at different frequencies. Reasonable predictions are obtained for the polarization hysteretic responses at all electric field amplitudes and frequencies. At lower frequencies some mismatches between the prediction and experimental data of the strains are observed, which might be due to the delayed time dependent response from poling the fibers prior to applying cyclic electric field. 
Figure 8 and Figure 9 show that as electric field amplitude increases, the mismatches between prediction and experiment also increase. We believe this is due to the possible nonlinear effect as the electric field increases. Linear model is typically valid when the amplitude of external stimuli is relatively small, and increasing the amplitude of external stimuli often leads to departure from a linear response. The proposed model is derived for linear time-dependent electro-mechanical response, and by linear we mean that the responses satisfy proportionality (linearity) and superposition conditions. For example some nonlinear effect that can occur in the material is that at higher electric field, the material can creep or relax at faster rate compared to when the electric field is relatively small. This type of accelerated time-dependent effect cannot be captured by linear model. Our intention in showing the responses at relatively high electric field $(0.5 \mathrm{MV} / \mathrm{m})$ to illustrates the departure of the responses from linear behavior. 

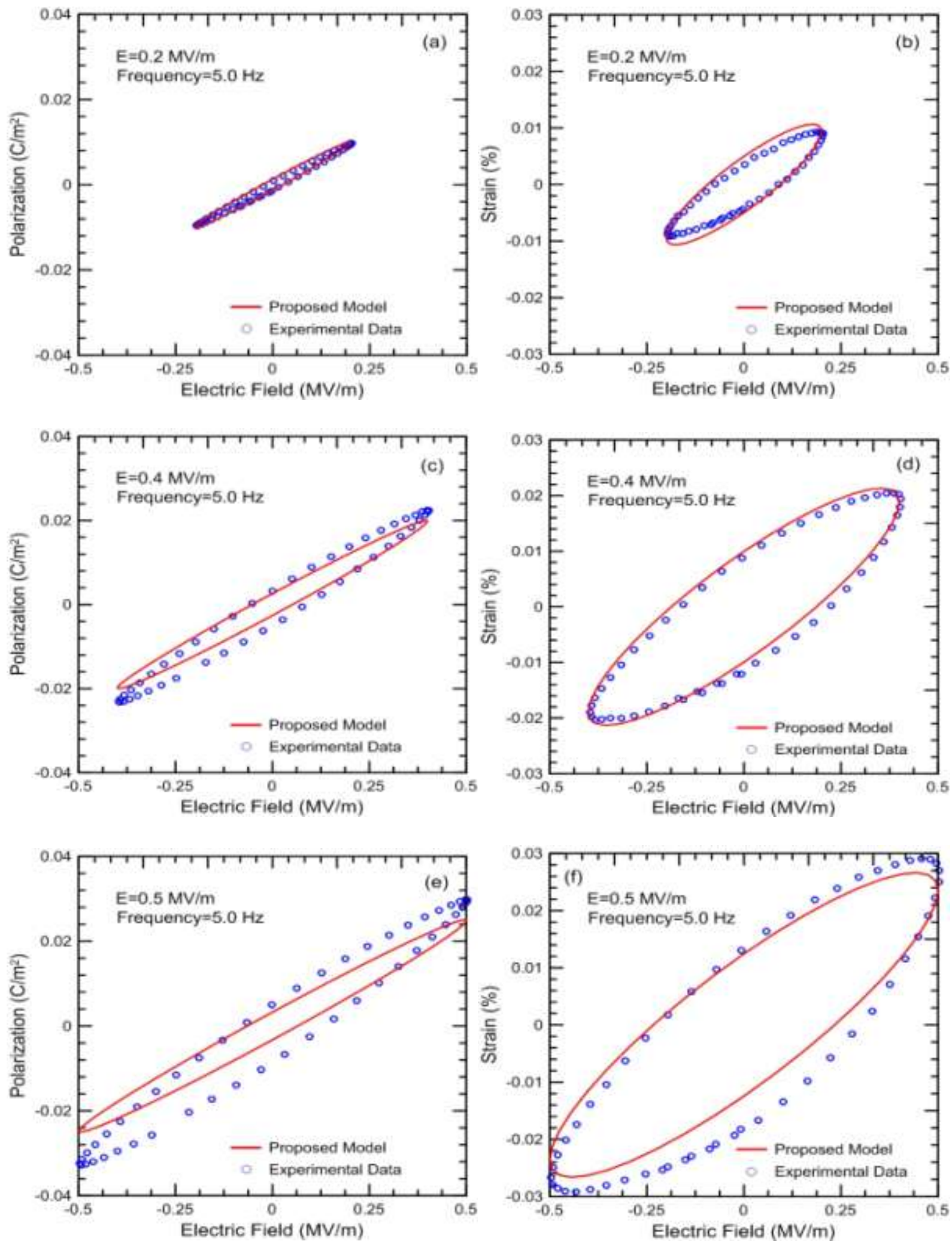

Figure 8 Predicted and experimental curve for time dependent hysteresis a), c),e) electric field vs polarization b), d), f) electric field vs strain behavior of PZT fiber at calibrated frequency. 

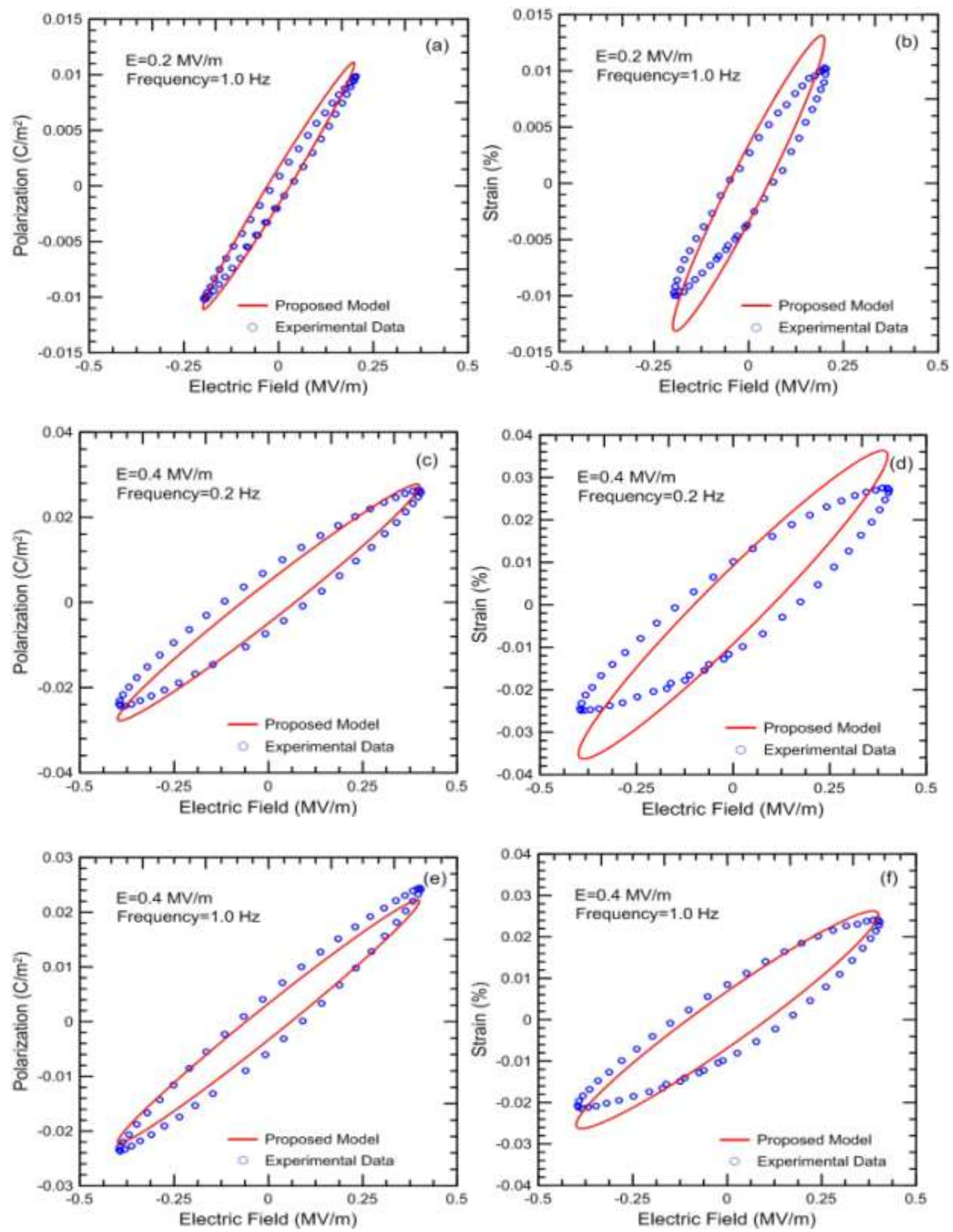

Figure 9 Predicted and experimental curve for time dependent hysteresis a),c),e) electric field vs polarization b), d), f) electric field vs strain behavior of PZT fiber at non-calibrated frequency. 
c) Homogeneous AFC

We calibrated the time dependent piezoelectric coupling parameters of AFC. The polarization and strain hysteretic responses under electric field of $E_{3}=0.61 \mathrm{MV} / \mathrm{m}$ at different frequencies were considered. The effective time dependent strain constants $\Delta d_{33}^{M E}(t)$ and $\Delta \kappa_{33}^{\sigma}(t)$ are characterized using Equations (46)-(47) when the stress is set to zero. Figure 10 shows the experimental data and calibrated response of the electric field and strain hysteretic responses under cyclic electric field of $E_{3}=0.61 \mathrm{MV} / \mathrm{m}$ at $1 \mathrm{~Hz}$. Table 7 shows the corresponding Prony series coefficients for time dependent kernel function given in Eq. (32). The instantaneous time-independent material properties and other physical properties are also given for the AFC.

Table 7 Prony series coefficients, mechanical, electromechanical and physical properties of AFC.

\begin{tabular}{|l|l|l|l|l|}
\hline $\mathrm{NP}$ & ${ }^{I} \lambda_{33}^{M E}\left(\mathrm{sec}^{-1}.\right)$ & ${ }^{I} d_{33}^{M E}\left(10^{-12} \mathrm{~m} / \mathrm{V}\right)$ & ${ }^{I} \lambda_{33}^{\sigma}\left(\mathrm{sec}^{-1}.\right)$ & ${ }^{I} \kappa^{\sigma}{ }_{33}\left(10^{-9} \mathrm{~F} / \mathrm{m}\right)$ \\
\hline 1 & 0.2 & 10 & 0.4 & 110 \\
\hline 2 & 1.0 & 120 & 4 & 2 \\
\hline 3 & 10.0 & 10 & 5 & 10 \\
\hline 4 & 60.0 & 140 & 90 & 12 \\
\hline Specific heat, $(c)=1500 \mathrm{~J} / \mathrm{kg}^{\circ} \mathrm{K}$ \\
Density, $(\rho)=2200 \mathrm{Kg} / \mathrm{m}^{3}$ \\
Time independent Material Properties \\
Piezoelectric Charge Constants: $d_{33}^{M E}=110 \times 10^{-12} \mathrm{~m} / \mathrm{V}$. \\
Relative Permittivity Constants: $\kappa_{33}^{\sigma} / \kappa_{0}=750$ \\
\hline
\end{tabular}



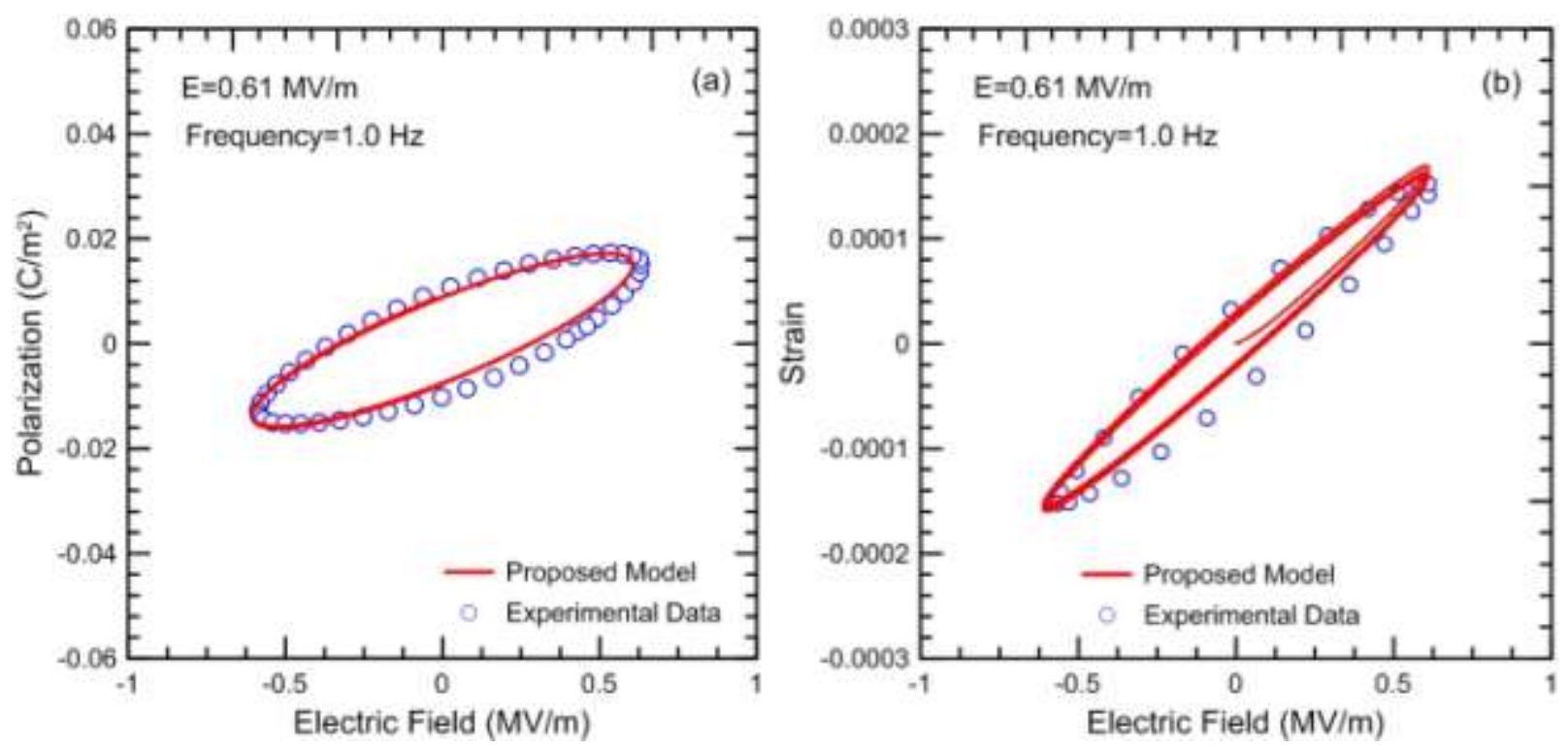

Figure 10 Calibrated and experimental curve for time dependent hysteresis a) electric field vs polarization b) electric field vs strain behavior of AFC at electric field (E3) of $0.61 \mathrm{MV} / \mathrm{m}$ and frequency of $\mathrm{f}=1.0 \mathrm{~Hz}$.

Next we predicted the hysteretic responses of AFC at different frequencies that were not used in the calibration process. The responses of electric field vs polarization (E-P) and electric field vs strain (E-S) curves at different frequencies are shown in Figure 11 (a)-(e). Pretty good predictions are observed which show the ability of the time-dependent model in capturing both hysteretic strains and polarizations responses of AFC under various frequencies. It is noted that the strain data at $10 \mathrm{~Hz}$ are missing. 

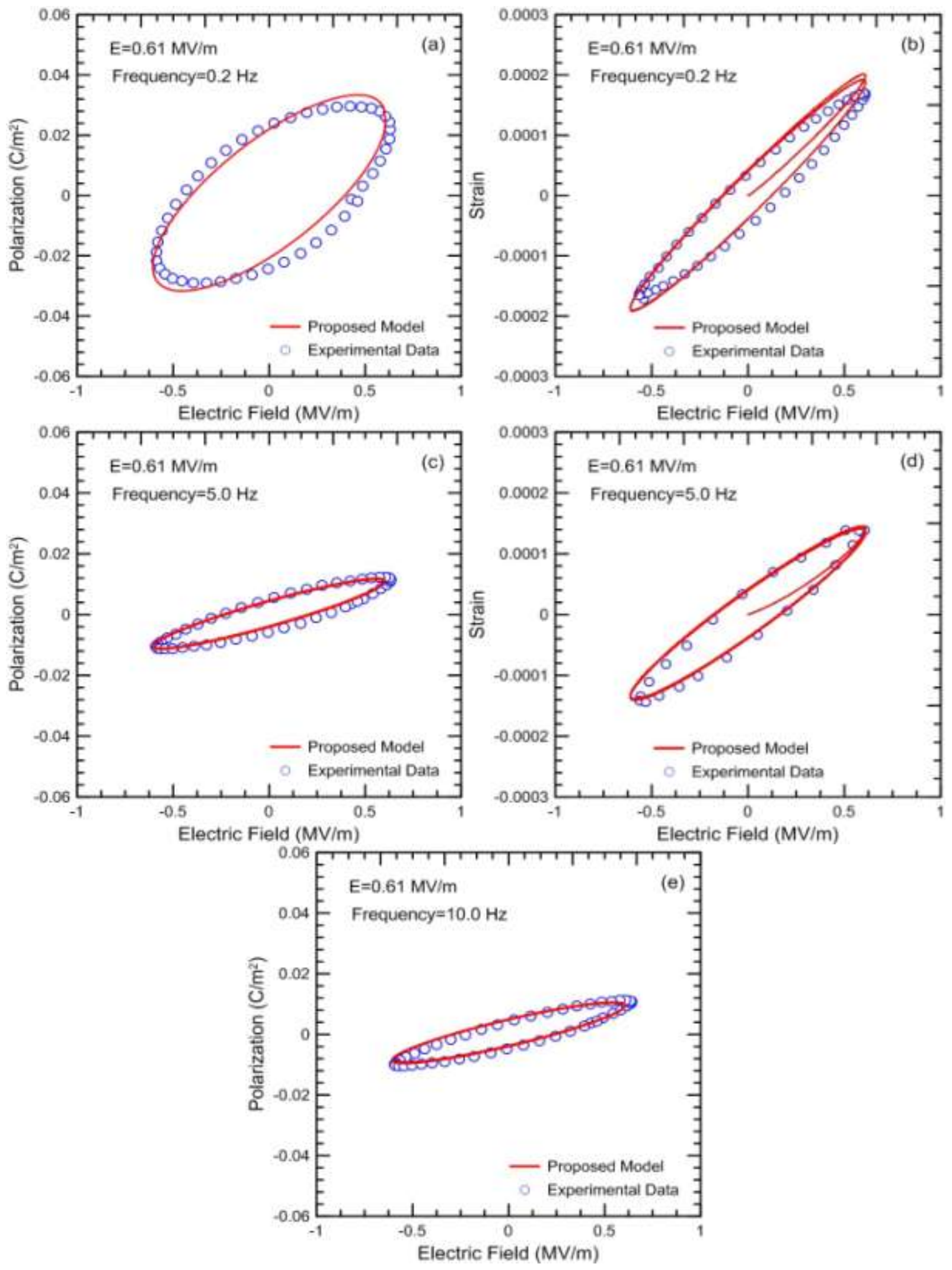

Figure 11 . Predicted and experimental curve for time dependent hysteresis a), c), e) electric field vs polarization b), d) electric field vs strain behavior of AFC at non-calibrated frequency. 


\subsection{Amount of heat generation during cyclic electric field inputs}

Using the calibrated properties, we studied the temperature rise due to the dissipation of energy under cyclic electric field in the different piezoelectric materials discussed above. We calculate the temperature rise in PZT fibers measured using the IDE method. Cyclic electric field is applied at different magnitude and frequencies for 600 seconds (10 minutes). Figure 12(a) shows the polarization and electric field hysteretic responses at different amplitude of electric field at a frequency of $1.0 \mathrm{~Hz}$. With the increase of amplitude the loop becomes wider, which increases the maximum polarization value and amount of energy being dissipated in one cycle. Figure 12 (b) (d) shows the temperature rise at different amplitude of electric field and at frequencies of 0.1, 1.0 and $10.0 \mathrm{~Hz}$. It can be seen that higher magnitude of electric field leads to higher temperature increases, and smaller temperature increases are observed at lower frequency compare to the ones at higher frequencies. This is because at higher frequencies, number of cycles after 10 minute loading is much higher than the one under low frequencies, accumulating more energy being dissipated. Unfortunately, measuring the temperature increase in PZT fibers due to cyclic electric field is very challenging as the dimensions of the fibers are very small, and immediate heat loss is observed during fiber testing. We, therefore, only measure temperature increase for AFC samples at different frequencies and amplitude, which is discussed later in this manuscript. 

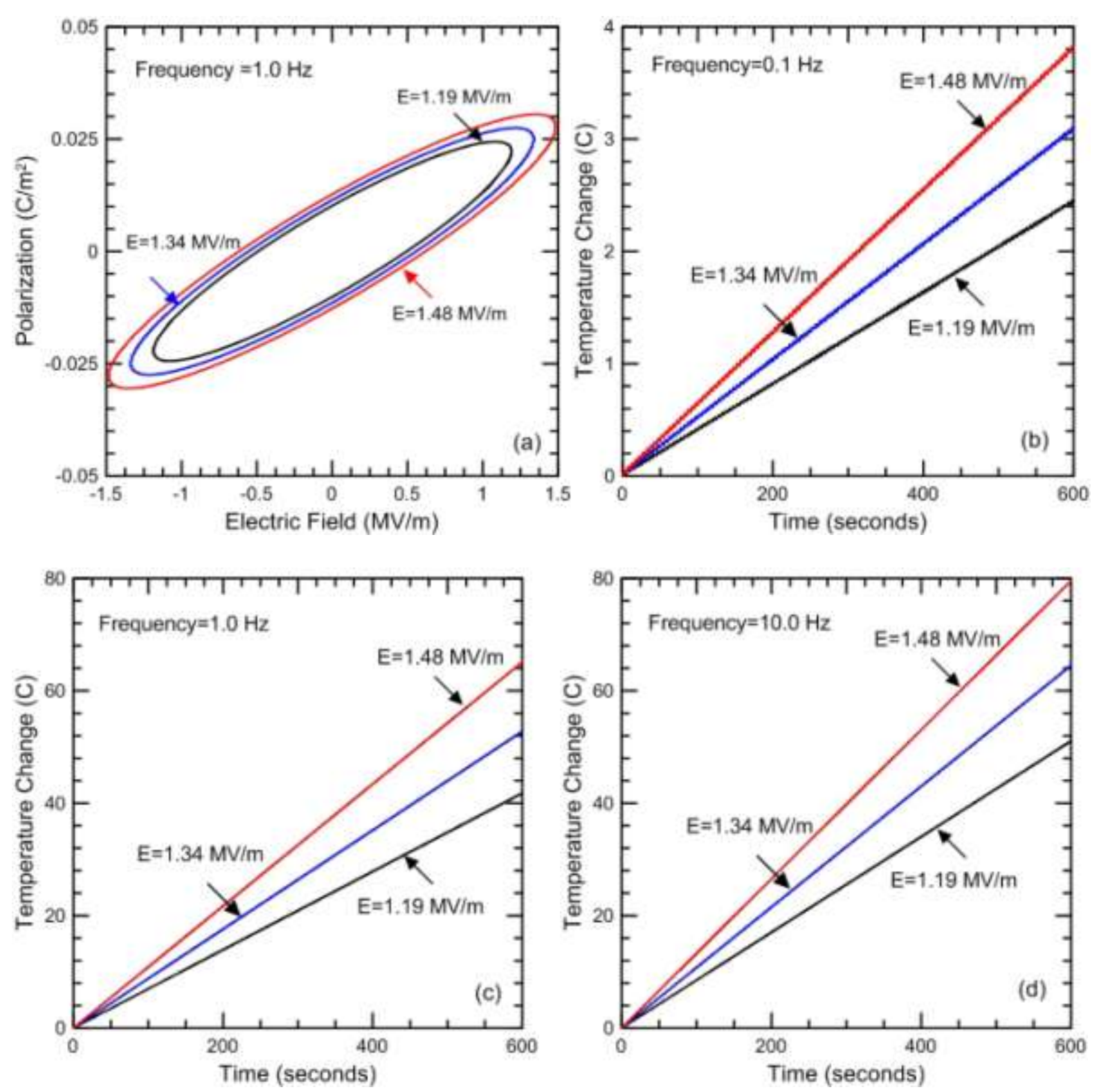

Figure 12 (a) Predicted polarization vs electric field hysteresis at different amplitude of electric field at frequency of $1.0 \mathrm{~Hz}$. Temperature rise in PZT fiber due to different electric field magnitude and at frequencies of (b) 0.1 , (c) 1.0 and (d) $10.0 \mathrm{~Hz}$

Similarly, we determine the temperature rise for PZT fiber characterized from parallel electrode setup at different amplitudes and frequencies. In this measurement, both polarization and strain responses were recorded. Cyclic electric field is applied at different magnitude and frequencies for 600 seconds (10 minutes). As shown in Eq. (49) the amount of energy dissipation 
due to cyclic electric field input is the combination of the energy dissipation from the hysteretic polarization and strain responses, i.e., electric field vs polarization (E-P) and electric field vs strain (E-S) curves. It is noted that experimentally we only recorded strains along the direction of electric field and we did not have any data on the transverse strain hysteretic response. In order to examine the contribution of energy dissipation from the polarization and strain hysteretic responses, we calculate the temperature increase independently and compare them. Figure 13 (a) and (b) shows the temperature rise from the E-P and E-S hysteresis at two extreme cases, i.e., 0.2 $\mathrm{MV} / \mathrm{m}$ at a frequency of $0.2 \mathrm{~Hz}$ and at $0.5 \mathrm{MV} / \mathrm{m}$ at a frequency of $5 \mathrm{~Hz}$, respectively. It is seen that the E-P hysteresis generates much higher energy dissipation, reflected in higher temperature increase, compared to the ones due to E-S hysteresis. Thus ignoring the dissipation due to the transverse strain effect should not cause any significant error. Once again it is seen that higher frequency leads to higher temperature increases.
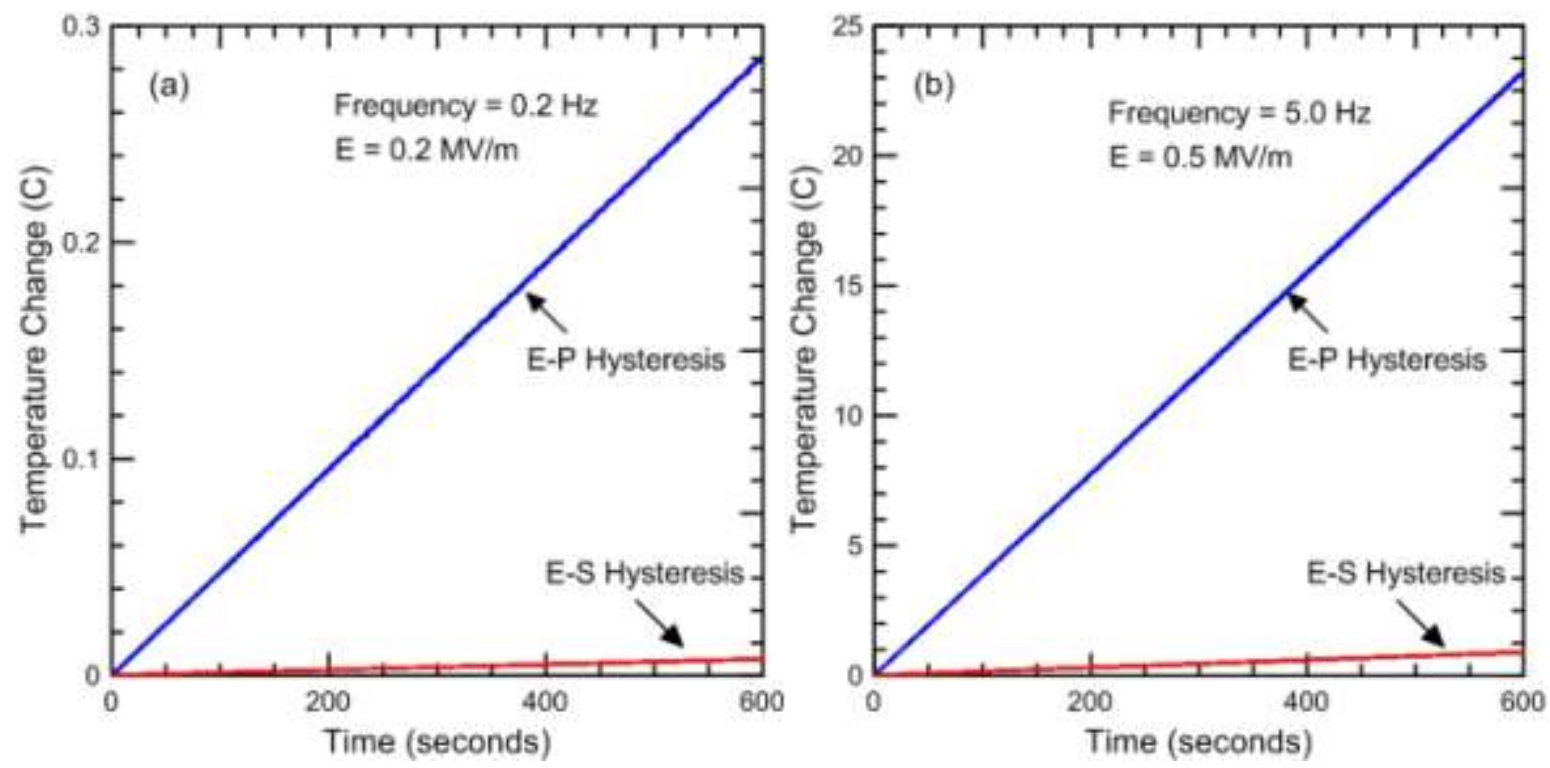

Figure 13 Predicted temperature rise in fiber at different electric field and frequencies.

Next, we compare the temperature rise for AFC due to both E-P and E-S hysteresis responses. Cyclic electric field is applied at magnitude of $E_{3}=0.61,1.19$. and $1.48 \mathrm{MV} / \mathrm{m}$ and at 
different frequencies $f=0.1$ and $10 \mathrm{~Hz}$. Figure 14 and Figure 15 show the temperature rise in AFC at frequencies 0.1 and $10 \mathrm{~Hz}$, respectively. Predictions from the model with the calibrated properties of AFC in Table 7 are given in dashed lines, and comparison with responses of PZT fiber is also shown with solid lines. Experimental data are shown with symbols. It is seen that at higher frequency and higher amplitude, more energy is being dissipated which translates in higher temperature increases. With regards to the effect of frequency and amplitude of electric field, the model predictions and experimental results are consistent, except for the experimental data under electric field amplitude of $0.61 \mathrm{MV} / \mathrm{m}$, in which temperature increases are about the same for both frequencies. Some discrepancies between the predictions and experiments are observed, which could be due to the following reasons: The model assumes adiabatic process, while in experiments convection heat transfer can occur in the specimens as testing was done in an open air condition with ambient temperature around $23^{\circ} \mathrm{C}$. It is noted that the experimental data show lower temperature increase at longer duration of loading, which in part could be due to heat loss. The linear electro-mechanical model is currently considered and the material parameters are assumed constants. It is possible that temperature increase would affect the properties of AFC, especially for the polymeric components. The temperature increases show nonlinear behavior with loading period, while as expected the linear model show linear increase in temperature. It should be noted that we have compared the experimental data, in terms of temperature measurement on the surface of the specimens, with the adiabatic solution. Other forms of heat transfer may have also occurred during testing. However, measuring possible heat loss due to convection, and detailed heat conduction within specimens are challenging, which is beyond the current scope of this study. The focus of this study is to highlight the heat generation during cyclic loading, which is associated to the linear hysteretic responses of the material. 

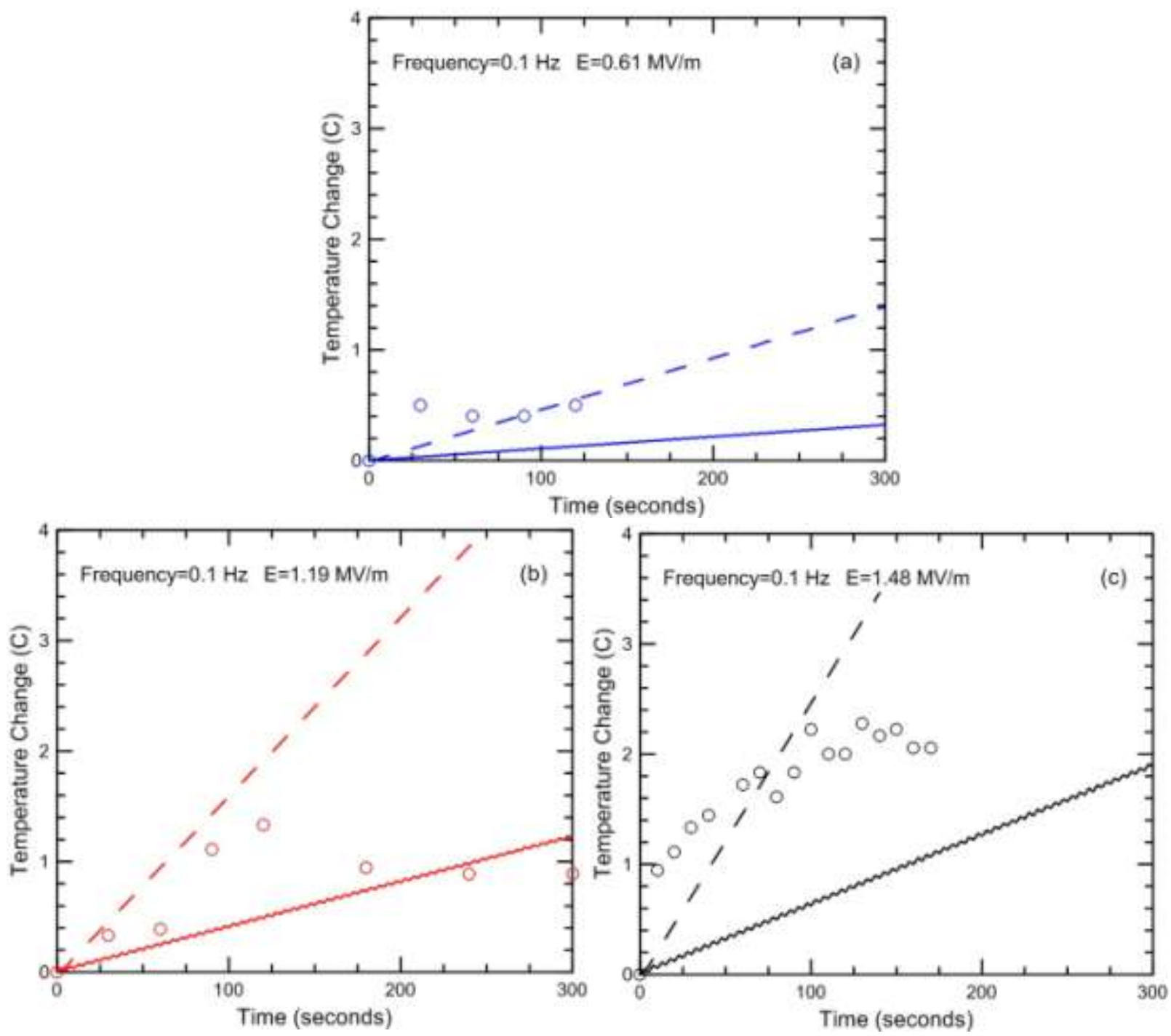

Figure 14 Predicted temperature rise in AFC at different amplitude and $\mathrm{f}=\mathbf{0 . 1} \mathrm{Hz}$ (solid lines are response of PZT, dashed lines represent responses from the model, and symbols denote experimental data). 

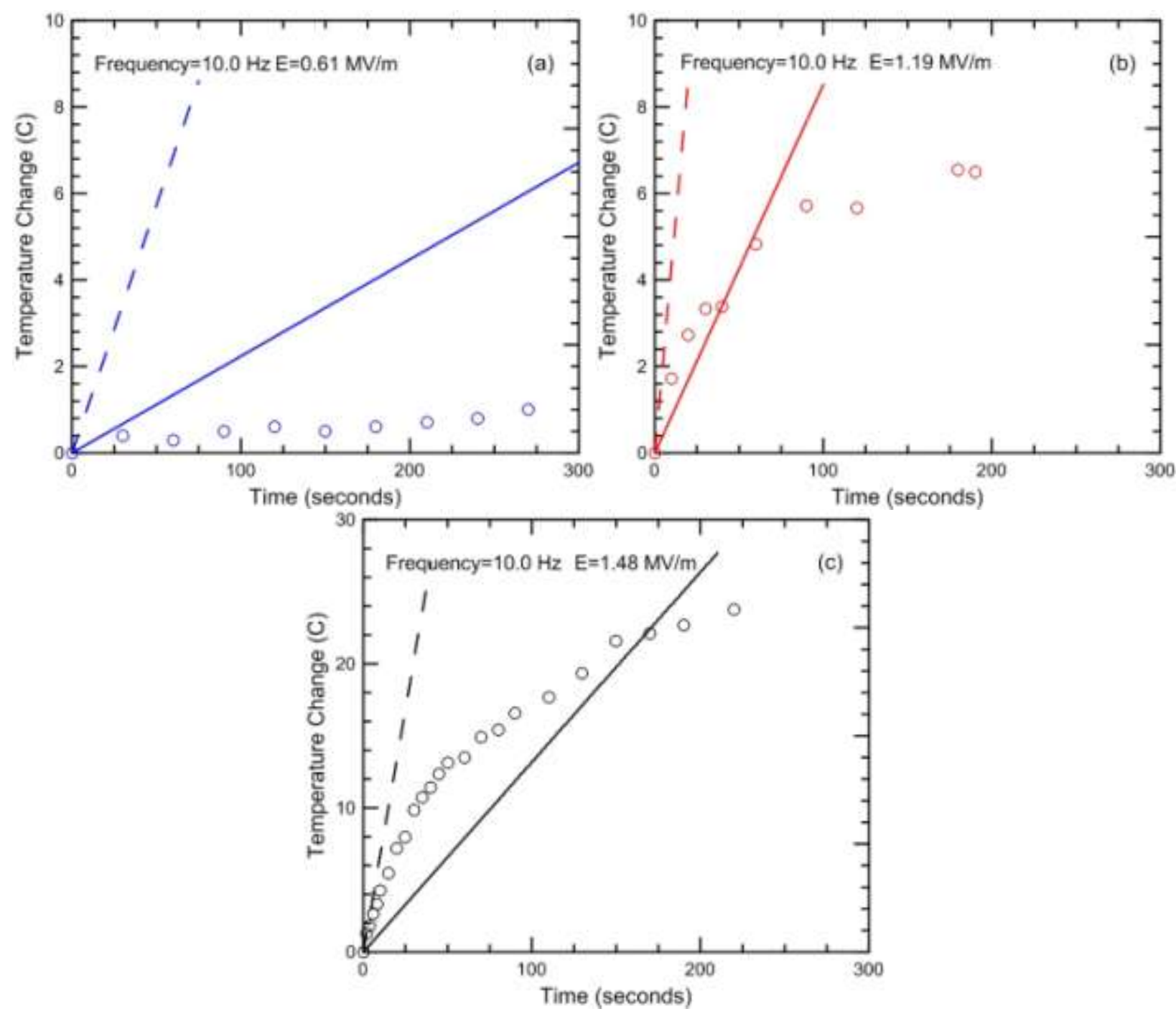

Figure 15 Predicted temperature rise in AFC at different amplitude and $f=10 \mathrm{~Hz}$ (solid lines are response of PZT, dashed lines represent responses of AFC, and symbols denote experimental data)

\section{Conclusions}

We developed a constitutive model for fully coupled electro thermo-viscoelastic response of piezoelectric based materials that takes into account the heat generation due to different dissipation mechanisms, namely the rate dependent viscous, electromechanical coupling and dielectric responses. Experimental tests on piezoelectric ceramics fibers and active fiber composites were conducted under cyclic electric fields at different frequencies and amplitude, 
below the coercive electric field limits of the materials. The constitutive model is shown to capture the hysteretic responses of these materials at various histories of loadings. We also studied the heat generation during linear hysteresis response under cyclic electric fields. It was observed that significant heat is generated from the dielectric relaxation mechanisms as compared to energy dissipated from electromechanical coupling mechanism. Heat generation was also found to be dependent on the magnitude and frequencies of electric field. The significant amount of temperature rise can alter the properties of soft PZT structures and thus affect their functionality. To avoid degradation of piezoelectric devices like actuators used in structures it is therefore necessary to estimate the temperature rise so that we can better predict the responses of these structures under combined electromechanical loadings.

\section{Acknowledgement}

This research is supported by the National Science Foundation Grant CMMI 1437437 and

CMMI-1437086 as part of a collaborative award between Penn State University and Texas A\&M University. 


\section{REFERENCES}

Atitallah, H.B., Ounaies, Z., Muliana, A., 2016. Temperature and time dependence of the electromechanical properties of flexible active fiber composites. Smart Mater. Struct. 25, 45002. doi:10.1088/0964-1726/25/4/045002

Ben Atitallah, H., Ounaies, Z., Muliana, A., 2010. Temperature and time effects in the electromechanical coupling behavior in active fiber composite, in: Proceedings of 16th US National Congress on Theoretical and Applied Mechanics USNCTAM, State College, Pennsylvania.

Bent, A.A., Hagood, N.W., 1995. Improved performance in piezoelectric fiber composites using interdigitated electrodes. pp. 196-212. doi:10.1117/12.209822

Chen, X., 2009. Nonlinear electro-thermo-viscoelasticity. Acta Mech. 211, 49-59. doi:10.1007/s00707-009-0217-9

Fett, T., Thun, G., 1998. Determination of Room-temperature Tensile Creep of PZT. J. Mater. Sci. Lett. 17, 1929-1931. doi:10.1023/A:1006608509876

Khan, K.A., Muliana, A.H., 2012. Fully coupled heat conduction and deformation analyses of visco-elastic solids. Mech. Time-Depend. Mater. 16, 461-489.

Li, J., Weng, G.J., 1999. A theory of domain switch for the nonlinear behaviour of ferroelectrics. Proc. R. Soc. Lond. Math. Phys. Eng. Sci. 455, 3493-3511. doi:10.1098/rspa.1999.0462

Lines, M.E., Glass, A.M., 1977. Principles and applications of ferroelectrics and related materials. Oxford university press.

Liu, Q.D., 2011. Investigation into the creeping polarization and strain in PZT-855 under combined mechanical and electrical loadings. Acta Mech. 220, 1-14. doi:10.1007/s00707-011-0462-6

Muliana, A., 2011. Time dependent behavior of ferroelectric materials undergoing changes in their material properties with electric field and temperature. Int. J. Solids Struct. 48, 2718-2731. doi:10.1016/j.ijsolstr.2011.05.021

Muliana, A., Khan, K.A., 2008. A time-integration algorithm for thermo-rheologically complex polymers. Comput. Mater. Sci. 41, 576-588.

Pritchard, J., Ramesh, R., Bowen, C.R., 2004. Time-temperature profiles of multi-layer actuators. Sens. Actuators Phys. 115, 140-145. doi:10.1016/j.sna.2004.04.019

Rajagopal, K.R., Srinivasa, A.R., 2011. A Gibbs-potential-based formulation for obtaining the response functions for a class of viscoelastic materials. Proc. R. Soc. Lond. Math. Phys. Eng. Sci. 467, 39-58. doi:10.1098/rspa.2010.0136

Schapery, R.A., 1997. Nonlinear Viscoelastic and Viscoplastic Constitutive Equations Based on Thermodynamics. Mech. Time-Depend. Mater. 1, 209-240. doi:10.1023/A:1009767812821

Schmidt, N.A., 1981. Coercive force and $90^{\circ}$ domain wall motion in ferroelectric PLZT ceramics with square hysteresis loops. Ferroelectrics 31, 105-111. doi:10.1080/00150198108201980

Tashiro, S., Ikehiro, M., Igarashi, H., 1997. Influence of Temperature Rise and Vibration Level on Electromechanical Properties of High-Power Piezoelectric Ceramics. Jpn. J. Appl. Phys. 36, 3004-3009. doi:10.1143/JJAP.36.3004

Truesdell, C., Noll, W., 2013. The Non-Linear Field Theories of Mechanics/Die Nicht-Linearen Feldtheorien der Mechanik. Springer Science \& Business Media.

Uchino, K., 2010. Advanced Piezoelectric Materials: Science and Technology. Elsevier. 
Uchino, K., Hirose, S., 2001. Loss mechanisms in piezoelectrics: how to measure different losses separately. IEEE Trans. Ultrason. Ferroelectr. Freq. Control 48, 307-321. doi:10.1109/58.896144

Ye, Z.-G. (Ed.), 2008. Handbook of Advanced Dielectric, Piezoelectric and Ferroelectric Materials: Synthesis, Properties and Applications, 1 edition. ed. Woodhead Publishing, Cambridge.

Zheng, J., Takahashi, S., Yoshikawa, S., Uchino, K., de Vries, J.W.C., 1996. Heat Generation in Multilayer Piezoelectric Actuators. J. Am. Ceram. Soc. 79, 3193-3198. doi:10.1111/j.1151-2916.1996.tb08095.x

Zhou, D., Kamlah, M., 2006. Room-temperature creep of soft PZT under static electrical and compressive stress loading. Acta Mater. 54, 1389-1396.

doi:10.1016/j.actamat.2005.11.010 